\title{
Variation Patterns of the Volatile Compounds in Flowers of Chinese Native Citrus Species and Their Taxonomic Implications
}

\author{
Wanpeng $\mathrm{Xi}^{1,3}$, Ligai $\mathrm{Li}^{2}$, Dong Jiang ${ }^{2}$, Bining $\mathrm{Jiao}^{2,4,5, *}$, Zhiqin Zhou ${ }^{1,3, *}$ \\ ${ }^{1}$ College of Horticulture and Landscape Architecture, Southwest University, Chongqing, China \\ ${ }^{2}$ Citrus Research Institute, Chinese Academy of Agricultural Sciences, Chongqing, China \\ ${ }^{3}$ Key Laboratory of Horticulture Science for Southern Mountainous Regions, Ministry of Education, Chongqing, China \\ ${ }^{4}$ National Citrus Engineering Research Center, Chongqing, China \\ ${ }^{5}$ Laboratory of Quality \& Safety Risk Assessment for Citrus Products (Chongqing), Ministry of Agriculture, Citrus Research Institute, \\ Southwest University, Chongqing, China \\ *Corresponding author: bljiao@tom.com; zqzhou2013@swu.edu.cn
}

Received March 24, 2015; Revised April 04, 2015; Accepted April 08, 2015

\begin{abstract}
In this study, the volatile compounds in the flowers of nine Citrus species/varieties, seven of which are native to China, were analyzed using headspace-solid phase microextraction (HS-SPME) coupled with gas chromatography mass spectrometry (GC-MS). A total of 94 compounds were identified, including various terpenes, such as monoterpenes, sesquiterpenes, terpene alcohols and aldehydes, which together accounted for $80.4 \%$ to $92.4 \%$ of the total compounds analyzed. Limonene, linalool and $\gamma$-terpinene were the dominant terpenes. Different species/varieties were characterized by their volatile compounds. Papeda was characterized by a high level of $\beta$ ocimene, linalyl acetate, myrcene and neo-alloocimene; Citrophorum was characterized by a high level of limonene and caryophyllene, and Cephelocitrus by a high level of limonene, $\beta$-pinene and linolool. Sinocitrus had the highest amount of linolool. Sweet orange had the highest level of limonene, while sour orange was distinct from others with the highest level of $\gamma$-terpinene. The four basic types of the genus Citrus L., Papeda, Cephalocitrus, Citrophorum and Sinocitrus, can be clearly classified based on a cluster analysis of their volatile compounds. All of the presumed hybrid species, including Jinchengbeibei 447 (C. sinensis Osb.), Goutoucheng (C. aurantium L.), Ningmeng 4 (C. limon Burm.f.), and Changshanhuyou (C. paradisi cv. Changshanhuyou), were grouped closely together with a suggested parent species in the constructed dendrogram. Our study clearly demonstrates that Citrus flower volatile compounds and their variation patterns can be used for Citrus species identification and taxonomic study.
\end{abstract}

Keywords: Citrus, volatile compounds, variation patterns, chemotaxonomy genus

Cite This Article: Wanpeng Xi, Ligai Li, Dong Jiang, Bining Jiao, and Zhiqin Zhou, "Variation Patterns of the Volatile Compounds in Flowers of Chinese Native Citrus Species and Their Taxonomic Implications." Journal of Food and Nutrition Research, vol. 3, no. 4 (2015): 235-245. doi: 10.12691/jfnr-3-4-2.

\section{Introduction}

Citrus is one of the most important fruit crops in the world (Hwang et al. 2012). Citrus fruits are popular food items because of their beautiful colors, pleasant flavors, and rich nutritional components (Tripoli et al. 2007). Citrus fruits can be consumed fresh or be processed into desserts, juices and jams and are good resources of vitamin C, folic acid, flavonoids, dietary fiber and many other health-promoting substances (Miller et al. 2011). In the food industry, citrus processing produces an enormous amount of byproducts, such as pomace, which represents $50 \%$ of the raw fruits and is a good source of volatile compounds (Anwar et al. 2008; Omori et al. 2011). In addition, citrus essential oils, which are a mixture of volatile compounds consisting mainly of monoterpene hydrocarbons, have been widely used in food, pharmaceutical and cosmetic industries as flavoring agents, and as antibacterial, antifungal and antioxidant agents (Ferhat et al. 2006).

The volatile compounds of cultivated citrus species have been widely studied. Fisher and Phillips reported that the basic components of citrus volatiles were terpenes, aldehydes, ketones, alcohols, esters and other compounds (Fisher et al. 2008). Jabalpurwala et al. (2009) compared the blossom volatile compounds of grapefruit, sour orange, sweet orange, lemon, mandarin, pumelo and lime and found that the citrus volatile profiles are primarily quantitative. Flamini et al. (2007) compared the volatiles of the different organs of Citrus lemon and found that young leaves have higher amount of limonene than the old ones. In addition, the chemical polymorphisms in the blossom oils of Citrus aurantium var. amara cultivated in different provinces were analyzed (Boussaada et al. 2007; Liu et al. 2013). Chung (2012) compared the volatile compounds of liquid essential oils and volatiles of the 
Hallabong blossom with two different fibers (CAR/PDMS and PAMS fibers). From the reports mentioned above, we found that many factors, such as genotype, province, season, developmental stage, extraction and analytical methods, influence the compositions of citrus volatiles (Liu et al. 2013; Cheong et al. 2011; González-Mas et al. 2011; Vekiari et al. 2001).

China is the most significant origin of the genus Citrus L. (Cheong et al. 2011). More than 4000 years of history of citrus cultivation have been recorded in China, and many important citrus species/varieties native to China have been described, such as Citrus ichangesis Swingle, Citrus grandis Osbeck, Citrus reticulata Blanco and Citrus aurantium L. (Al-Ababneh et al. 2002; Zhang et al. 2004; Spiegel et al. 1996). The bioactive components in Papeda, the texture and flavor of Guangxishatianyou, the color of Taiwanpenggan, and the resistance to stress of Citrus aurantium L. of these native Chinese germplasms have been investigated in previous studies (Spiegel et al. 1996). Citrus leaves, blossoms, fruit and the exocarp of some species, such as Citrus aurantiu L., Citrus medica L. and Citrus grandis (L.) Osbeck, have traditionally been used in Chinese medicines for their anti-inflammatory, cough relief and lung-purging properties (Lu et al. 2006). Despite the great economic and medicinal potential for citrus plants, information regarding the volatile components of various wild Citrus species, especially those native to China, is still limited (Jabalpurwala et al.
2009; González-Mas et al. 2011; Fanciullino et al. 2006; Hosni et al. 2010).

In this study, in an attempt to analyze the influence of genetic and/or evolutionary factors on the composition and contents of citrus volatile components, we analyze the volatile compounds in the flowers of basic Citrus types and their presumed hybrids, which are mostly represented by the genotypes of Chinese origin. We also explore the influence of hybridization on the variation of Citrus volatile compounds. The results provide useful information for future germplasm utilization and the taxonomy of the Citrus genus.

\section{Materials and Methods}

\subsection{Plant Material}

Blossoming flowers were collected from trees cultivated at the China National Citrus Germplasm Repository, Citrus Research Institute of Chinese Academy of Agricultural Sciences, Chongqing, China. For each species/variety, three trees were selected. All three of the basic species of the subgenus Eucitrus of Swingle's system, C. medica L., C. grandis Osbeck., C. reticulata Blanco. and Papeda were represented. The voucher specimens were returned to the Citrus Research Institute of Chinese Academy of Agricultural Sciences (CRIC), Chongqing, China. The information about the samples is given in detail in Table 1.

Table 1. The Citrus species analyzed in the present study

\begin{tabular}{|c|c|c|c|c|c|c|}
\hline NO. ${ }^{a}$ & Citrus species & Cutivars $^{\mathrm{b}}$ & Abbreviation & Section name & National Unified number & Repository number ${ }^{\mathrm{b}}$ \\
\hline 1 & Citrus sinensis. Osbeck & Jinchengbeibei 447 & JCBB & Aurantium & LS0306 & GPGJ0573 \\
\hline 2 & C. aurantium L. & Goutoucheng & GTC & Aurantium & LA0008 & GPGJ0011 \\
\hline 3 & C. ichangensis Swingle & Yichangcheng & YCC & Papeda & LP0067 & GPGJ0701 \\
\hline 4 & C. reticulata Blanco & Edanhongju & EDHJ & Sinocitrus & LR0040 & GPGJ0059 \\
\hline 5 & C. reticulata Blanco & Taiwanpenggan & TWPG & Sinocitrus & LR0658 & GPGJ1093 \\
\hline 6 & C. grandis Osbeck & Guangxishatianyou & GXSTY & Cephalocitrus & LG0016 & GPGJ0100 \\
\hline 7 & C. paradis Macf. & Changshanhuyou & $\mathrm{CSHY}$ & Cephalocitrus & LG0181 & GPGJ0645 \\
\hline 8 & C. limon (L). Burm.f. & Ningmeng 4 & SHNM & Citrophorum & LM0183 & GPGJ1001 \\
\hline 9 & C. medica $\mathrm{L}$ & Dannaxaingyuan & DNXY & Citrophorum & LM0026 & GPGJ0539 \\
\hline
\end{tabular}

${ }^{\mathrm{a}}$ Number of sample

${ }^{\mathrm{b}}$ Repository Number of the National Citrus Germplasm Repository, Chongqing, China.

\subsection{Isolation and Concentration of Volatiles}

Freshly collected flowers were taken to the laboratory immediately, washed using deionized water and patted dry with gauze. The cleaned flowers were immediately ground into powder and stored at $-80^{\circ} \mathrm{C}$ until use. The isolation and concentration of flower volatiles was carried out by headspace-solid phase microextraction (HS-SPME) method. For each extraction, $1.5 \mathrm{~g}$ of the powder, $3 \mathrm{ml}$ of a saturated solution of sodium chloride and $2 \mu \mathrm{L}$ of cyclohexanone (added as an internal standard) were added to a 20-mL capped vial. The sealed vials were analyzed by HS-SPME. A temperature of $50^{\circ} \mathrm{C}$ was selected for preincubation and headspace extraction based on a previous study (Liu et al. 2013). Pre-incubation and extraction times were $20 \mathrm{~min}$ and $50 \mathrm{~min}$, respectively. Desorption was performed for $5 \mathrm{~min}$ at $250{ }^{\circ} \mathrm{C}$ in splitless mode. All experiments were performed in triplicate.

\subsection{GC-MS Conditions}

The GC-MS analyses were carried out using an Agilent 7890A gas chromatograph coupled to a flame ionization detector and a 5975C single quadrupole mass detector. A DB-5MS silica capillary column $(30 \mathrm{~m} \times 0.25 \mathrm{~mm} \times 0.25 \mu \mathrm{m}$ film thickness) was used. The initial temperature of column oven was held constant at $35^{\circ} \mathrm{C}$ for $5 \mathrm{~min}$, followed by an increase to $180^{\circ} \mathrm{C}$ at $3^{\circ} \mathrm{C} \mathrm{min}^{-1}$ ramp and was held constant at $250^{\circ} \mathrm{C}$ for $10 \mathrm{~min}$. The temperature was then increased to $240^{\circ} \mathrm{C}$ at $5^{\circ} \mathrm{C} \mathrm{min}{ }^{-1}$ (held for $2 \mathrm{~min}$ ). Helium was used as the carrier gas at $1 \mathrm{~mL} \mathrm{~min}^{-1}$ constant flow rate, and the mass spectrometer operated in the electron impact (EI) mode (ionization energy, $70 \mathrm{eV}$; source temperature $230^{\circ} \mathrm{C}$ ). Full scanning mode was used for the data acquisition, and the mass range was $\mathrm{m} / \mathrm{z}$ 35400 . 
Table 2. The chemical compositions and their contents of in the flower volatiles of the Citrus species analyzed.

\begin{tabular}{|c|c|c|c|c|c|c|c|c|c|c|c|c|c|}
\hline \multirow{2}{*}{ No. } & \multirow{2}{*}{$\mathrm{RI}^{\mathrm{a}}$} & \multirow{2}{*}{$\mathrm{CAS}^{\mathrm{b}}$} & \multirow{2}{*}{ Compounds } & \multirow{2}{*}{ Codes $^{c}$} & \multicolumn{9}{|c|}{ Genotypes $^{\mathrm{d}, \mathrm{f}, \mathrm{g}}$} \\
\hline & & & & & JCBB & GTC & YCC & EDHJ & TWPG & GXSTY & CSHY & SHNM & DNXY \\
\hline \multicolumn{14}{|c|}{ Monoterpenes } \\
\hline 1 & 993 & $\begin{array}{l}123- \\
35-3\end{array}$ & myrcene & M1 & $\begin{array}{c}396.3 \pm \\
18.8\end{array}$ & $\begin{array}{c}369.3 \pm \\
21.4\end{array}$ & $\begin{array}{c}542.9 \pm \\
32.1\end{array}$ & $\begin{array}{c}158.4 \pm \\
9.84\end{array}$ & $\begin{array}{c}488.3 \pm \\
39.2\end{array}$ & $90.4 \pm 7.2$ & $\begin{array}{c}112.5 \pm \\
6.56\end{array}$ & $\begin{array}{c}348.6 \pm \\
32.5\end{array}$ & nd \\
\hline 2 & 925 & $\begin{array}{c}2867- \\
5-2\end{array}$ & $\alpha$-thujene & M2 & nd & $\begin{array}{c}52.11 \pm \\
3.21\end{array}$ & nd & $\begin{array}{c}20.03 \pm \\
1.56\end{array}$ & $\begin{array}{c}118.9 \pm \\
8.23\end{array}$ & $\begin{array}{c}3.56 \pm \\
0.31\end{array}$ & nd & $\begin{array}{c}10.53 \pm \\
1.26\end{array}$ & nd \\
\hline 3 & 929 & $\begin{array}{c}7785- \\
26-4\end{array}$ & (-)- $\alpha$-pinene & M3 & $\begin{array}{c}22.3 \pm \\
1.06\end{array}$ & nd & nd & nd & nd & nd & nd & nd & $\begin{array}{c}61.94 \pm \\
4.88\end{array}$ \\
\hline 4 & 930 & $\begin{array}{c}80-56- \\
8\end{array}$ & $\alpha$-pinene & M4 & $\begin{array}{c}64.39 \pm \\
2.98\end{array}$ & $\begin{array}{c}256.6 \pm \\
17.5\end{array}$ & nd & $\begin{array}{c}57.19 \pm \\
4.55\end{array}$ & $\begin{array}{c}156.1 \pm \\
9.54\end{array}$ & $\begin{array}{c}67.31 \pm \\
5.15\end{array}$ & $\begin{array}{c}66.21 \pm \\
4.69\end{array}$ & $\begin{array}{c}203.2 \pm \\
18.4\end{array}$ & nd \\
\hline 5 & 932 & $\begin{array}{c}99-83- \\
2\end{array}$ & $\alpha$-phellandrene & M5 & $\begin{array}{c}26.04 \pm \\
3.15\end{array}$ & $\begin{array}{c}252.8 \pm \\
19.1\end{array}$ & nd & nd & $\begin{array}{c}32.36 \pm \\
2.06\end{array}$ & nd & $\begin{array}{c}10.47 \pm \\
0.84\end{array}$ & nd & $\begin{array}{c}4.71 \pm \\
0.25\end{array}$ \\
\hline 6 & 944 & $\begin{array}{c}79-92- \\
5\end{array}$ & camphene & M6 & $\begin{array}{c}2.78 \pm \\
0.39\end{array}$ & $\begin{array}{c}3.79 \pm \\
0.18\end{array}$ & nd & nd & nd & $\begin{array}{c}7.29 \pm \\
5.16\end{array}$ & nd & $\begin{array}{c}13.74 \pm \\
2.15\end{array}$ & nd \\
\hline 7 & 975 & $\begin{array}{l}127- \\
91-3\end{array}$ & $\beta$-pinene & M7 & $\begin{array}{c}3.79 \pm \\
0.26\end{array}$ & $\begin{array}{c}322.1 \pm \\
18.2\end{array}$ & nd & nd & nd & $\begin{array}{c}954.2 \pm \\
69.8\end{array}$ & $\begin{array}{c}325.7 \pm \\
26.5\end{array}$ & $\begin{array}{c}1629 \pm \\
89.3\end{array}$ & nd \\
\hline 8 & 976 & $\begin{array}{c}3387- \\
41-5\end{array}$ & sabinene & M8 & nd & nd & nd & nd & nd & nd & nd & nd & nd \\
\hline 9 & 982 & $\begin{array}{c}18172- \\
67-3\end{array}$ & (-)- $\beta$-pinene & $\mathrm{M}^{\mathrm{e}}$ & nd & nd & nd & $\begin{array}{c}94.07 \pm \\
6.25\end{array}$ & nd & nd & nd & nd & $\begin{array}{c}144.4 \pm \\
9.8\end{array}$ \\
\hline 10 & 998 & $\begin{array}{l}460- \\
01-5\end{array}$ & cosmene & $\mathrm{M} 10^{\mathrm{e}}$ & nd & nd & $\begin{array}{c}13.81 \pm \\
1.23\end{array}$ & nd & nd & $\begin{array}{c}10.83 \pm \\
1.65\end{array}$ & nd & nd & nd \\
\hline 11 & 1016 & $\begin{array}{c}99-86- \\
5\end{array}$ & $\alpha$-terpinene & M11 & $\begin{array}{c}93.67 \pm \\
6.91\end{array}$ & $\begin{array}{c}53.37 \pm \\
4.91\end{array}$ & $\begin{array}{c}2.44 \pm \\
0.25\end{array}$ & $\begin{array}{c}14.13 \pm \\
2.12\end{array}$ & $\begin{array}{c}147.3 \pm \\
9.84\end{array}$ & $\begin{array}{c}6.87 \pm \\
0.58\end{array}$ & nd & $\begin{array}{c}1.51 \pm \\
0.01\end{array}$ & $\begin{array}{c}4.05 \pm \\
0.26\end{array}$ \\
\hline 12 & 1021 & $\begin{array}{c}99-87- \\
6\end{array}$ & p-cymene & M12 & nd & $\begin{array}{c}469.2 \pm \\
23.3\end{array}$ & nd & $\begin{array}{c}97.8 \pm \\
6.35\end{array}$ & $\begin{array}{c}188.2 \pm \\
9.21\end{array}$ & nd & $\begin{array}{c}154.9 \pm \\
9.2\end{array}$ & $\begin{array}{c}5.29 \pm \\
0.38\end{array}$ & nd \\
\hline 13 & 1029 & $\begin{array}{l}138- \\
86-3\end{array}$ & limonene & M13 & $\begin{array}{c}1016 \pm \\
59.8\end{array}$ & $\begin{array}{c}436 \pm \\
25.3\end{array}$ & $\begin{array}{c}60.91 \pm \\
4.53\end{array}$ & $\begin{array}{c}268.8 \pm \\
18.9\end{array}$ & $\begin{array}{c}382.8 \pm \\
35.2\end{array}$ & $\begin{array}{c}1073 \pm \\
65.4\end{array}$ & $\begin{array}{c}268.6 \pm \\
19.4\end{array}$ & $\begin{array}{c}5650 \pm \\
145\end{array}$ & $\begin{array}{c}5224 \pm \\
89.1\end{array}$ \\
\hline 14 & 1039 & $\begin{array}{c}3338- \\
55-4\end{array}$ & (z)-ocimene & M14 & $\begin{array}{c}410.1 \pm \\
25.6\end{array}$ & $\begin{array}{c}10.93 \pm \\
1.45\end{array}$ & nd & $\begin{array}{c}6.24 \pm \\
0.42\end{array}$ & nd & $\begin{array}{c}43.99 \pm \\
5.21\end{array}$ & nd & $\begin{array}{c}171.2 \pm \\
13.2\end{array}$ & $\begin{array}{c}210.9 \pm \\
19.5\end{array}$ \\
\hline 15 & 1044 & $\begin{array}{c}13877- \\
91-3\end{array}$ & $\beta$-ocimene & $\mathrm{M} 15^{\mathrm{e}}$ & $\begin{array}{c}3.79 \pm \\
0.29\end{array}$ & $\begin{array}{c}924.3 \pm \\
56.6\end{array}$ & $\begin{array}{c}1940 \pm \\
98.5\end{array}$ & $\begin{array}{c}831.9 \pm \\
75.6\end{array}$ & $\begin{array}{c}1304 \pm \\
56.4\end{array}$ & nd & $\begin{array}{c}346.5 \pm \\
26.8\end{array}$ & nd & $\begin{array}{c}532.4 \pm \\
17.8\end{array}$ \\
\hline 16 & 1055 & $\begin{array}{c}3779- \\
61-1\end{array}$ & (e)-ocimene & M16 & nd & nd & $\begin{array}{c}396.5 \pm \\
26.9\end{array}$ & nd & nd & $\begin{array}{c}1173 \pm \\
105.2\end{array}$ & nd & $\begin{array}{c}1173 \pm \\
96.4\end{array}$ & nd \\
\hline 17 & 1060 & $\begin{array}{c}13466- \\
78-9\end{array}$ & 3-carene & M17 & $\begin{array}{c}42.83 \pm \\
3.61\end{array}$ & nd & $\begin{array}{c}19.33 \pm \\
3.11\end{array}$ & nd & nd & nd & nd & $\begin{array}{c}23.47 \pm \\
2.19\end{array}$ & nd \\
\hline 18 & 1064 & $\begin{array}{c}99-85- \\
4\end{array}$ & $\gamma$-terpinene & M18 & $\begin{array}{c}151.4 \pm \\
9.7\end{array}$ & $\begin{array}{c}1591 \pm \\
105\end{array}$ & $\begin{array}{c}1.44 \pm \\
0.21\end{array}$ & $\begin{array}{c}428.6 \pm \\
32.5\end{array}$ & $\begin{array}{c}847.5 \pm \\
85.3\end{array}$ & $\begin{array}{c}16.66 \pm \\
0.85\end{array}$ & $\begin{array}{c}920.1 \pm \\
56.8\end{array}$ & $\begin{array}{c}735.5 \pm \\
14.2\end{array}$ & $\begin{array}{c}258.8 \pm \\
14.5\end{array}$ \\
\hline 19 & 1088 & $\begin{array}{l}586- \\
62-9\end{array}$ & $\delta$-terpinene & M19 & $\begin{array}{c}52.14 \pm \\
3.71\end{array}$ & nd & $\begin{array}{c}19.25 \pm \\
2.15\end{array}$ & nd & nd & $\begin{array}{c}11.7 \pm \\
1.23\end{array}$ & $\begin{array}{c}68.57 \pm \\
4.15\end{array}$ & $\begin{array}{c}66.56 \pm \\
4.56\end{array}$ & nd \\
\hline 20 & 1131 & $\begin{array}{c}7216- \\
56-0\end{array}$ & neo-alloocimene & M20 & $\begin{array}{c}29.49 \pm \\
2.94\end{array}$ & $\begin{array}{c}45.31 \pm \\
3.15\end{array}$ & $\begin{array}{c}364.3 \pm \\
15.4\end{array}$ & $\begin{array}{c}47.35 \pm \\
4.11\end{array}$ & $\begin{array}{c}56.83 \pm \\
3.25\end{array}$ & $\begin{array}{c}57.72 \pm \\
6.35\end{array}$ & $\begin{array}{c}14.15 \pm \\
2.11\end{array}$ & $\begin{array}{c}171.1 \pm \\
8.22\end{array}$ & $\begin{array}{c}66.99 \pm \\
4.56\end{array}$ \\
\hline & & & Total & & $\begin{array}{c}3925 \pm \\
209\end{array}$ & $\begin{array}{c}4787 \pm \\
196\end{array}$ & $\begin{array}{c}3367 \pm \\
205\end{array}$ & $\begin{array}{c}2024 \pm \\
147\end{array}$ & $\begin{array}{c}5533 \pm \\
213\end{array}$ & $\begin{array}{c}3516 \pm \\
236\end{array}$ & $2288 \pm 89$ & $\begin{array}{c}10203 \pm \\
369\end{array}$ & $\begin{array}{c}6508 \pm \\
185\end{array}$ \\
\hline & & & Relatives (\%) & & $61.33 \%$ & $60.35 \%$ & $53.05 \%$ & $38.04 \%$ & $51.52 \%$ & $54.60 \%$ & $53.89 \%$ & $82.59 \%$ & $57.00 \%$ \\
\hline \multicolumn{14}{|c|}{ Sesqueterpenes } \\
\hline 21 & 1336 & $\begin{array}{c}20307- \\
84-0\end{array}$ & elemene & $\mathrm{S} 1$ & nd & $\begin{array}{c}97.97 \pm \\
6.15\end{array}$ & $\begin{array}{c}22.75 \pm \\
3.21\end{array}$ & $\begin{array}{c}63.57 \pm \\
1.97\end{array}$ & $\begin{array}{c}70.73 \pm \\
4.05\end{array}$ & $\begin{array}{c}35.59 \pm \\
2.38\end{array}$ & $\begin{array}{c}27.77 \pm \\
1.66\end{array}$ & $\begin{array}{c}27.96 \pm \\
3.09\end{array}$ & nd \\
\hline 22 & 1348 & $\begin{array}{c}17699- \\
14-8\end{array}$ & $\alpha$-cubebene & $\mathrm{S} 2$ & $\begin{array}{c}2.78 \pm \\
0.36\end{array}$ & $\begin{array}{c}10.97 \pm \\
1.56\end{array}$ & $\begin{array}{c}77.18 \pm \\
5.61\end{array}$ & $\begin{array}{c}3.41 \pm \\
0.48\end{array}$ & $\begin{array}{c}4.05 \pm \\
0.51\end{array}$ & $\begin{array}{c}1.88 \pm \\
0.24\end{array}$ & $\begin{array}{c}3.94 \pm \\
0.28\end{array}$ & $\begin{array}{c}1.15 \pm \\
0.24\end{array}$ & $\begin{array}{c}22.1 \pm \\
1.46\end{array}$ \\
\hline 23 & 1374 & $\begin{array}{c}3856- \\
25-5\end{array}$ & copaene & S3 & $\begin{array}{c}7.29 \pm \\
0.82\end{array}$ & $\begin{array}{c}19.98 \pm \\
2.03\end{array}$ & $\begin{array}{c}229.7 \pm \\
15.6\end{array}$ & $\begin{array}{c}6.31 \pm \\
0.31\end{array}$ & $\begin{array}{c}7.25 \pm \\
0.56\end{array}$ & $\begin{array}{c}1.75 \pm \\
0.24\end{array}$ & nd & $\begin{array}{c}1.07 \pm \\
0.13\end{array}$ & $\begin{array}{c}15.16 \pm \\
1.22\end{array}$ \\
\hline 24 & 1383 & $\begin{array}{c}5208- \\
59-3\end{array}$ & $\beta$-bourbonene & $\mathrm{S} 4^{\mathrm{e}}$ & nd & nd & $\begin{array}{c}26.6 \pm \\
0.31\end{array}$ & nd & nd & nd & nd & nd & nd \\
\hline 25 & 1391 & $\begin{array}{l}515- \\
13-9\end{array}$ & $\beta$-elemene & S5 & $\begin{array}{c}103.4 \pm \\
8.2\end{array}$ & $\begin{array}{c}30.77 \pm \\
2.96\end{array}$ & $\begin{array}{c}41.85 \pm \\
3.03\end{array}$ & $\begin{array}{c}27.44 \pm \\
1.47\end{array}$ & $\begin{array}{c}34.98 \pm \\
2.65\end{array}$ & $\begin{array}{c}9.85 \pm \\
1.07\end{array}$ & $\begin{array}{c}160.9 \pm \\
10.6\end{array}$ & $\begin{array}{c}2.48 \pm \\
0.25\end{array}$ & nd \\
\hline 26 & 1406 & $\begin{array}{c}17699- \\
05-7\end{array}$ & $\alpha$-bergamotene & S6 & $\begin{array}{c}10.9 \pm \\
1.06\end{array}$ & $\begin{array}{c}9.58 \pm \\
1.23\end{array}$ & $\begin{array}{c}2.14 \pm \\
0.15\end{array}$ & nd & $\begin{array}{c}25.4 \pm \\
2.33\end{array}$ & nd & $\begin{array}{c}11.93 \pm \\
0.84\end{array}$ & $\begin{array}{c}152.3 \pm \\
9.14\end{array}$ & $\begin{array}{c}535.7 \pm \\
25.5\end{array}$ \\
\hline 27 & 1419 & $\begin{array}{c}87-44- \\
5\end{array}$ & caryophyllene & S7 & $\begin{array}{c}92.09 \pm \\
6.53\end{array}$ & $\begin{array}{c}299.6 \pm \\
35.6\end{array}$ & $\begin{array}{c}552.9 \pm \\
63.6\end{array}$ & $\begin{array}{c}69.95 \pm \\
4.54\end{array}$ & $\begin{array}{c}73.9 \pm \\
5.71\end{array}$ & $\begin{array}{c}219.2 \pm \\
18.3\end{array}$ & $\begin{array}{c}160.1 \pm \\
10.7\end{array}$ & $\begin{array}{c}414.3 \pm \\
19.6\end{array}$ & $\begin{array}{c}897.8 \pm \\
69.8\end{array}$ \\
\hline 28 & 1427 & $\begin{array}{c}13744- \\
15-5\end{array}$ & $\beta$-cubebene & S8 & nd & $\begin{array}{c}9.65 \pm \\
1.24\end{array}$ & $\begin{array}{c}9.82 \pm \\
1.02\end{array}$ & $\begin{array}{c}2.88 \pm \\
0.18\end{array}$ & $\begin{array}{c}5.78 \pm \\
0.41\end{array}$ & $\begin{array}{c}2.71 \pm \\
0.36\end{array}$ & $\begin{array}{c}5.33 \pm \\
0.29\end{array}$ & nd & $\begin{array}{c}17.8 \pm \\
1.57\end{array}$ \\
\hline 29 & 1433 & $\begin{array}{l}33915 \\
4-91-5\end{array}$ & $\gamma$-elemene & S9 & nd & $\begin{array}{c}72.63 \pm \\
8.62\end{array}$ & $\begin{array}{c}55.71 \pm \\
4.76\end{array}$ & $\begin{array}{c}62.13 \pm \\
8.27\end{array}$ & $\begin{array}{c}68.32 \pm \\
4.36\end{array}$ & $\begin{array}{c}8.51 \pm \\
1.07\end{array}$ & $\begin{array}{c}53.8 \pm \\
0.67\end{array}$ & nd & nd \\
\hline 30 & 1436 & $\begin{array}{c}13474- \\
59-4\end{array}$ & trans- $\alpha$-bergamotene & $\mathrm{S} 10$ & nd & nd & $40.95 \pm$ & nd & nd & nd & nd & nd & nd \\
\hline
\end{tabular}




\begin{tabular}{|c|c|c|c|c|c|c|c|c|c|c|c|c|c|}
\hline \\
\hline 31 & 1439 & $\begin{array}{c}3691- \\
12-1\end{array}$ & $\alpha$-guaiene & $\mathrm{S} 11^{\mathrm{e}}$ & nd & nd & $\begin{array}{c}12.7 \pm \\
1.08\end{array}$ & nd & nd & nd & $\begin{array}{c}2.77 \pm \\
0.32\end{array}$ & nd & nd \\
\hline 32 & 1441 & $\begin{array}{c}25246- \\
27-9\end{array}$ & l-alloaromadendrene & $\mathrm{S} 12^{\mathrm{e}}$ & nd & $\begin{array}{c}14.17 \pm \\
2.13\end{array}$ & nd & $\begin{array}{c}15.92 \pm \\
1.36\end{array}$ & nd & nd & nd & $\begin{array}{c}0.92 \pm \\
0.13\end{array}$ & nd \\
\hline 33 & 1451 & $\begin{array}{l}6753- \\
98-6\end{array}$ & $\alpha$-caryophyllene & $\mathrm{S} 13^{\mathrm{e}}$ & $\begin{array}{c}15.59 \pm \\
1.36\end{array}$ & $\begin{array}{c}32.53 \pm \\
2.98\end{array}$ & $\begin{array}{c}74.32 \pm \\
8.16\end{array}$ & $\begin{array}{c}14.11 \pm \\
1.29\end{array}$ & $\begin{array}{c}14.41 \pm \\
0.97\end{array}$ & $\begin{array}{c}20.96 \pm \\
1.56\end{array}$ & $\begin{array}{c}34.55 \pm \\
3.05\end{array}$ & $\begin{array}{c}30.9 \pm \\
2.38\end{array}$ & $\begin{array}{c}71.53 \pm \\
6.28\end{array}$ \\
\hline 34 & 1458 & $\begin{array}{c}18794- \\
84-8\end{array}$ & $\beta$-farnesene & $\mathrm{S} 14$ & $\begin{array}{c}146.2 \pm \\
10.2\end{array}$ & nd & nd & nd & nd & $\begin{array}{c}9.31 \pm \\
1.12\end{array}$ & $\begin{array}{c}193.2 \pm \\
15.7\end{array}$ & $\begin{array}{c}9.32 \pm \\
1.03\end{array}$ & $\begin{array}{c}19.82 \pm \\
3.21\end{array}$ \\
\hline 35 & 1460 & $\begin{array}{c}\text { 28973- } \\
97-9\end{array}$ & (z)- $\beta$-farnesene & $\mathrm{S} 15^{\mathrm{e}}$ & nd & $\begin{array}{c}253.3 \pm \\
19.1\end{array}$ & $\begin{array}{c}46.59 \pm \\
6.31\end{array}$ & $\begin{array}{c}1.54 \pm \\
0.18\end{array}$ & $\begin{array}{c}356.5 \pm \\
26.3\end{array}$ & nd & nd & $\begin{array}{c}6.33 \pm \\
0.54\end{array}$ & $\begin{array}{c}19.51 \pm \\
1.45\end{array}$ \\
\hline 36 & 1475 & $\begin{array}{c}30021- \\
74-0\end{array}$ & $\gamma$-muurolene & $\mathrm{S} 16^{\mathrm{e}}$ & nd & nd & $\begin{array}{c}41.91 \pm \\
3.16\end{array}$ & $\begin{array}{c}5.33 \pm \\
0.41\end{array}$ & $\begin{array}{c}4.76 \pm \\
0.39\end{array}$ & $\begin{array}{c}2.39 \pm \\
0.27\end{array}$ & $\begin{array}{c}6.89 \pm \\
0.81\end{array}$ & $\begin{array}{c}1.53 \pm \\
0.22\end{array}$ & $\begin{array}{c}11.01 \pm \\
0.96\end{array}$ \\
\hline 37 & 1480 & $\begin{array}{c}\text { 37839- } \\
63-7\end{array}$ & germacrene $\mathrm{d}$ & S17 & nd & $\begin{array}{c}59.46 \pm \\
3.69\end{array}$ & $\begin{array}{c}27.24 \pm \\
1.87\end{array}$ & $\begin{array}{c}18.59 \pm \\
2.34\end{array}$ & $\begin{array}{c}25.34 \pm \\
2.99\end{array}$ & $\begin{array}{c}12.62 \pm \\
1.97\end{array}$ & $\begin{array}{c}34.81 \pm \\
4.56\end{array}$ & nd & $\begin{array}{c}77.75 \pm \\
8.22\end{array}$ \\
\hline 38 & 1493 & $\begin{array}{l}473- \\
13-2\end{array}$ & (-)- $\alpha$-selinene & $\mathrm{S} 18^{\mathrm{e}}$ & $\begin{array}{c}1.87 \pm \\
0.25\end{array}$ & nd & $\begin{array}{c}18.05 \pm \\
2.13\end{array}$ & nd & nd & nd & $\begin{array}{c}17.72 \pm \\
0.54\end{array}$ & nd & nd \\
\hline 39 & 1495 & $\begin{array}{c}3242- \\
8-8\end{array}$ & elixene & $\mathrm{S} 19^{\mathrm{e}}$ & nd & nd & nd & $\begin{array}{c}40.35 \pm \\
3.62\end{array}$ & $\begin{array}{c}53.69 \pm \\
4.58\end{array}$ & $\begin{array}{c}19.43 \pm \\
2.55\end{array}$ & nd & $\begin{array}{c}29.94 \pm \\
1.67\end{array}$ & $\begin{array}{c}29.66 \pm \\
3.11\end{array}$ \\
\hline 40 & 1506 & $\begin{array}{c}3691- \\
11-0\end{array}$ & $\delta$-guaiene & $\mathrm{S} 20^{\mathrm{e}}$ & nd & nd & $\begin{array}{c}92.62 \pm \\
7.54\end{array}$ & nd & nd & nd & nd & nd & nd \\
\hline 41 & 1511 & $\begin{array}{l}495- \\
61-4\end{array}$ & l- $\beta$-bisabolene & $\mathrm{S} 21$ & nd & nd & $\begin{array}{c}193.3 \pm \\
23.8\end{array}$ & $\begin{array}{c}48.97 \pm \\
6.57\end{array}$ & nd & nd & nd & $\begin{array}{c}149.4 \pm \\
6.5\end{array}$ & $\begin{array}{c}586.9 \pm \\
37.7\end{array}$ \\
\hline 42 & 1512 & $\begin{array}{c}26560- \\
14-5\end{array}$ & $\alpha$-farnesene & $\mathrm{S} 22$ & $\begin{array}{c}32.99 \pm \\
4.12\end{array}$ & nd & nd & nd & $\begin{array}{c}110.2 \pm \\
9.85\end{array}$ & $\begin{array}{c}26.12 \pm \\
3.24\end{array}$ & $\begin{array}{c}4.81 \pm \\
0.57\end{array}$ & nd & nd \\
\hline 43 & 1522 & $\begin{array}{l}483- \\
76-1\end{array}$ & $\delta$-cadinene & $\mathrm{S} 23$ & $\begin{array}{c}15.63 \pm \\
2.14\end{array}$ & $\begin{array}{c}41.25 \pm \\
2.98\end{array}$ & nd & $\begin{array}{c}13.47 \pm \\
1.07\end{array}$ & nd & $\begin{array}{c}4.28 \pm \\
0.31\end{array}$ & $\begin{array}{c}21.62 \pm \\
3.88\end{array}$ & $\begin{array}{c}0.31 \pm \\
0.05\end{array}$ & $\begin{array}{c}31.48 \pm \\
4.15\end{array}$ \\
\hline 44 & 1523 & $\begin{array}{c}20307- \\
83-9\end{array}$ & $\beta$-sesquiphellandrene & S24 & nd & nd & nd & nd & $\begin{array}{c}37.45 \pm \\
4.15\end{array}$ & nd & nd & nd & nd \\
\hline 45 & 1525 & $\begin{array}{c}\text { 54324- } \\
03-7\end{array}$ & $\begin{array}{c}\text { (+)-epi- } \\
\text { bicyclosesquiphellandrene }\end{array}$ & $\mathrm{S} 25^{\mathrm{e}}$ & nd & nd & nd & nd & nd & nd & nd & nd & nd \\
\hline 46 & 1537 & $\begin{array}{c}4630- \\
07-3\end{array}$ & $(+)$-valencene & $\mathrm{S} 26^{\mathrm{e}}$ & nd & nd & nd & nd & nd & nd & nd & nd & $\begin{array}{c}15.35 \pm \\
1.47\end{array}$ \\
\hline \multirow[t]{4}{*}{47} & 1742 & $\begin{array}{c}10486- \\
19-8\end{array}$ & valencene & S27 & nd & nd & nd & nd & nd & nd & nd & nd & $\begin{array}{c}17.8 \pm \\
0.82\end{array}$ \\
\hline & & & Total & & $\begin{array}{c}428.7 \pm \\
56.4\end{array}$ & $\begin{array}{c}1003 \pm \\
84.2\end{array}$ & $\begin{array}{c}1677 \pm \\
205\end{array}$ & $\begin{array}{c}395.2 \pm \\
78.7\end{array}$ & $\begin{array}{c}892.8 \pm \\
54.1\end{array}$ & $\begin{array}{c}375.8 \pm \\
29.8\end{array}$ & $\begin{array}{c}740.2 \pm \\
58.6\end{array}$ & $\begin{array}{c}827.7 \pm \\
54.1\end{array}$ & $\begin{array}{c}2369 \pm \\
145\end{array}$ \\
\hline & & & Relatives (\%) & & $6.70 \%$ & $12.65 \%$ & $26.42 \%$ & $7.43 \%$ & $8.31 \%$ & $5.84 \%$ & $17.43 \%$ & $6.70 \%$ & $20.75 \%$ \\
\hline & \multicolumn{13}{|c|}{ Terpene Alcohols } \\
\hline 48 & 1068 & $\begin{array}{c}7299- \\
41-4\end{array}$ & cis- $\beta$-terpineol & $\mathrm{TA} 1^{\mathrm{e}}$ & $\begin{array}{c}22.31 \pm \\
3.27\end{array}$ & $\begin{array}{c}1.74 \pm \\
0.19\end{array}$ & nd & nd & $\begin{array}{c}45.49 \pm \\
3.67\end{array}$ & 1.26 & nd & $\begin{array}{c}5.03 \pm \\
0.37\end{array}$ & nd \\
\hline 49 & 1102 & $\begin{array}{l}78-70- \\
6\end{array}$ & linalool & TA2 & $\begin{array}{c}1097 \pm \\
127\end{array}$ & $\begin{array}{c}1198 \pm \\
105\end{array}$ & $\begin{array}{c}34.65 \pm \\
4.31\end{array}$ & $\begin{array}{c}2099 \pm \\
175\end{array}$ & $\begin{array}{c}2793 \pm \\
308\end{array}$ & $\begin{array}{c}1160 \pm \\
159\end{array}$ & $\begin{array}{c}489.3 \pm \\
24.3\end{array}$ & $\begin{array}{c}129.8 \pm \\
15.8\end{array}$ & $\begin{array}{c}64.97 \pm \\
5.14\end{array}$ \\
\hline 50 & 1124 & $\begin{array}{c}1960- \\
12-8\end{array}$ & phenethyl alcohol & $\mathrm{TA} 3^{\mathrm{e}}$ & $\begin{array}{c}10.27 \pm \\
2.15\end{array}$ & $\begin{array}{c}1.33 \pm \\
0.23\end{array}$ & nd & $\begin{array}{c}10.41 \pm \\
2.15\end{array}$ & nd & nd & $\begin{array}{c}17.43 \pm \\
2.65\end{array}$ & nd & nd \\
\hline 51 & 1176 & $\begin{array}{c}20126- \\
76-5\end{array}$ & (-)-4-terpineol & $\mathrm{TA} 4^{\mathrm{e}}$ & nd & $\begin{array}{c}11.07 \pm \\
1.45\end{array}$ & nd & nd & $\begin{array}{c}82.01 \pm \\
6.24\end{array}$ & nd & $\begin{array}{c}4.16 \pm \\
0.37\end{array}$ & $\begin{array}{c}13.47 \pm \\
1.65\end{array}$ & nd \\
\hline 52 & 1188 & $\begin{array}{l}562- \\
74-3\end{array}$ & 4-terpineol & TA5 & $\begin{array}{c}46.79 \pm \\
2.15\end{array}$ & nd & nd & $\begin{array}{c}5.19 \pm \\
0.41\end{array}$ & nd & nd & nd & nd & $\begin{array}{c}12.13 \pm \\
1.57\end{array}$ \\
\hline 53 & 1189 & $\begin{array}{c}10482- \\
56-1\end{array}$ & $\alpha$-terpineol & TA6 & $\begin{array}{c}61.61 \pm \\
2.39\end{array}$ & $\begin{array}{c}57.88 \pm \\
4.71\end{array}$ & $\begin{array}{c}2.66 \pm \\
0.29\end{array}$ & $\begin{array}{c}11.18 \pm \\
1.67\end{array}$ & $\begin{array}{c}132.9 \pm \\
13.8\end{array}$ & $\begin{array}{c}6.19 \pm \\
0.34\end{array}$ & $\begin{array}{c}7.69 \pm \\
0.64\end{array}$ & $\begin{array}{c}55.78 \pm \\
6.71\end{array}$ & $\begin{array}{c}57.5 \pm \\
3.66\end{array}$ \\
\hline 54 & 1218 & $\begin{array}{c}1197- \\
06-4\end{array}$ & cis-carveol & TA7 & nd & $\begin{array}{c}1.39 \pm \\
0.31\end{array}$ & nd & nd & nd & $\begin{array}{c}4.12 \pm \\
0.19\end{array}$ & nd & $\begin{array}{c}16.14 \pm \\
2.14\end{array}$ & $\begin{array}{c}44.38 \pm \\
3.25\end{array}$ \\
\hline 55 & 1295 & $\begin{array}{l}499- \\
75-2\end{array}$ & carvacrol & TA $8^{\mathrm{e}}$ & nd & $\begin{array}{c}83.02 \pm \\
6.34\end{array}$ & nd & $\begin{array}{c}165.9 \pm \\
12.3\end{array}$ & $\begin{array}{c}168.1 \pm \\
21.4\end{array}$ & $\begin{array}{c}0.25 \pm \\
0.04\end{array}$ & nd & nd & nd \\
\hline 56 & 1229 & $\begin{array}{l}106- \\
25-2\end{array}$ & nerol & TA9 & $\begin{array}{c}6.65 \pm \\
0.57\end{array}$ & $\begin{array}{c}2.61 \pm \\
0.32\end{array}$ & nd & nd & nd & $\begin{array}{c}148.4 \pm \\
16.7\end{array}$ & $\begin{array}{c}11.89 \pm \\
13.4\end{array}$ & $\begin{array}{c}17.75 \pm \\
2.11\end{array}$ & $\begin{array}{c}450.4 \pm \\
38.9\end{array}$ \\
\hline 57 & 1256 & $\begin{array}{l}106- \\
24-1\end{array}$ & geraniol & TA10 & $\begin{array}{c}1.55 \pm \\
0.17\end{array}$ & $\begin{array}{c}6.85 \pm \\
0.57\end{array}$ & nd & $\begin{array}{c}1.79 \pm \\
0.24\end{array}$ & $\begin{array}{c}12.31 \pm \\
1.95\end{array}$ & $\begin{array}{c}102.5 \pm \\
9.45\end{array}$ & $\begin{array}{c}14.08 \pm \\
2.07\end{array}$ & $\begin{array}{c}32.66 \pm \\
6.17\end{array}$ & $\begin{array}{c}345.3 \pm \\
28.3\end{array}$ \\
\hline 58 & 1565 & $\begin{array}{c}7212- \\
44-4\end{array}$ & nerolidol & TA11 & $\begin{array}{c}71.89 \pm \\
6.35\end{array}$ & nd & nd & $\begin{array}{c}7.57 \pm \\
0.87\end{array}$ & $\begin{array}{c}15.5 \pm \\
1.21\end{array}$ & $\begin{array}{c}195.2 \pm \\
23.6\end{array}$ & nd & $\begin{array}{c}55.38 \pm \\
4.36\end{array}$ & nd \\
\hline 59 & 1584 & $\begin{array}{l}142- \\
50-7\end{array}$ & d-nerolidol & TA12 & nd & nd & nd & nd & nd & nd & $\begin{array}{c}85.51 \pm \\
7.88\end{array}$ & nd & $\begin{array}{c}49.41 \pm \\
5.16\end{array}$ \\
\hline 60 & 1690 & $\begin{array}{c}20576- \\
54-9\end{array}$ & 2,3-dihydro-6-trans-farnesol & $\mathrm{TA}_{13}{ }^{\mathrm{e}}$ & nd & nd & nd & nd & nd & nd & nd & $\begin{array}{c}14.86 \pm \\
1.27\end{array}$ & $\begin{array}{c}28.29 \pm \\
3.34\end{array}$ \\
\hline 61 & 1731 & $\begin{array}{l}106- \\
28-5\end{array}$ & (e,e)-farnesol & TA14 & nd & nd & nd & nd & nd & nd & nd & $\begin{array}{c}17.1 \pm \\
1.69\end{array}$ & nd \\
\hline 62 & 1735 & $\begin{array}{c}4602- \\
84-0\end{array}$ & farnesol & TA15 & $6.33 \pm$ & nd & nd & nd & $1.55 \pm$ & $30.7 \pm$ & $18.83 \pm$ & $8.84 \pm$ & $27.61 \pm$ \\
\hline
\end{tabular}




\begin{tabular}{|c|c|c|c|c|c|c|c|c|c|c|c|c|c|}
\hline & & & & & 0.58 & & & & 0.37 & 2.19 & 3.12 & 0.97 & 4.22 \\
\hline & & & Total & & $1324 \pm$ & $1364 \pm$ & $37.31 \pm$ & $2301 \pm$ & $3251 \pm$ & $1649 \pm$ & $649.3 \pm$ & $367.2 \pm$ & $1080 \pm$ \\
\hline & & & IOtal & & 54.3 & 78.1 & 2.68 & 125 & 189 & 65.4 & 56.4 & 24.1 & 84.8 \\
\hline & & & Relatives (\%) & & $20.70 \%$ & $17.19 \%$ & $0.59 \%$ & $43.25 \%$ & $30.27 \%$ & $25.60 \%$ & $15.28 \%$ & $2.97 \%$ & $9.46 \%$ \\
\hline & & & & & Ter & ene Aldeh & ydes & & & & & & \\
\hline 63 & 1154 & $\begin{array}{l}106- \\
23-0\end{array}$ & citronellal & TD1 & $\begin{array}{c}4.46 \pm \\
0.57\end{array}$ & nd & nd & $\begin{array}{c}1.29 \pm \\
0.32\end{array}$ & $\begin{array}{c}17.17 \pm \\
2.15\end{array}$ & $\begin{array}{c}24.82 \pm \\
3.61\end{array}$ & $\begin{array}{c}8.55 \pm \\
0.95\end{array}$ & $\begin{array}{c}23.1 \pm \\
2.67\end{array}$ & $\begin{array}{c}58.71 \pm \\
4.94\end{array}$ \\
\hline 64 & 1221 & $\begin{array}{c}18031- \\
40-8\end{array}$ & l-perillaldehyde & $\mathrm{TD} 2^{\mathrm{e}}$ & $\begin{array}{c}2.91 \pm \\
0.27\end{array}$ & nd & nd & nd & $\begin{array}{c}14.56 \pm \\
1.22\end{array}$ & nd & nd & nd & nd \\
\hline 65 & 1243 & $\begin{array}{l}106- \\
26-3\end{array}$ & neral & TD3 & $\begin{array}{c}5.34 \pm \\
0.62\end{array}$ & $\begin{array}{c}1.33 \pm \\
0.07\end{array}$ & nd & nd & $\begin{array}{c}4.24 \pm \\
0.58\end{array}$ & $\begin{array}{c}173.4 \pm \\
21.5\end{array}$ & $\begin{array}{c}13.46 \pm \\
0.41\end{array}$ & $\begin{array}{c}164.3 \pm \\
16.7\end{array}$ & $\begin{array}{c}349.9 \pm \\
45.4\end{array}$ \\
\hline 66 & 1273 & $\begin{array}{l}141- \\
27-5\end{array}$ & geranial & TD4 & nd & nd & $\begin{array}{c}1.57 \pm \\
0.28\end{array}$ & nd & $\begin{array}{c}5.67 \pm \\
0.36\end{array}$ & nd & nd & nd & nd \\
\hline 67 & 1697 & $\begin{array}{c}4955- \\
32-2\end{array}$ & $\begin{array}{c}\text { 1,3,6,10-farnesatetraen-12- } \\
\text { al }\end{array}$ & $\mathrm{TD}^{\mathrm{e}}$ & $\begin{array}{c}7.15 \pm \\
0.67\end{array}$ & nd & nd & nd & $\begin{array}{c}10.35 \pm \\
1.23\end{array}$ & nd & nd & nd & nd \\
\hline & & & Total & & $19.86 \pm$ & $1.33 \pm$ & $1.57 \pm$ & $1.29 \pm$ & $51.99 \pm$ & $418.4 \pm$ & $39.25 \pm$ & $421.5 \pm$ & $408.6 \pm$ \\
\hline & & & tordit & & 2.19 & 0.18 & 0.16 & 0.08 & 6.7 & 32.5 & 4.7 & 26.4 & 33.8 \\
\hline & & & Relatives (\%) & & $0.31 \%$ & $0.02 \%$ & $0.02 \%$ & $0.02 \%$ & $0.48 \%$ & $6.50 \%$ & $0.92 \%$ & $3.41 \%$ & $3.58 \%$ \\
\hline & & & & & liphatic $A$ & dehydes A & ad Alcoho & & & & & & \\
\hline 68 & 798 & $\begin{array}{c}66-25- \\
1\end{array}$ & hexanal & $\mathrm{P} 1$ & $\begin{array}{c}85.52 \pm \\
5.69\end{array}$ & $\begin{array}{c}60.45 \pm \\
4.94\end{array}$ & $\begin{array}{c}42.27 \pm \\
3.61\end{array}$ & $\begin{array}{c}97.57 \pm \\
5.99\end{array}$ & $\begin{array}{c}96.75 \pm \\
6.37\end{array}$ & $\begin{array}{c}26.77 \pm \\
4.04\end{array}$ & $\begin{array}{c}101.5 \pm \\
9.31\end{array}$ & $\begin{array}{c}58.01 \pm \\
6.62\end{array}$ & $\begin{array}{c}91.41 \pm \\
8.08\end{array}$ \\
\hline 69 & 849 & $\begin{array}{l}6728- \\
26-3\end{array}$ & trans-2-hexenal & $\mathrm{P} 2^{\mathrm{e}}$ & $\begin{array}{c}230.2 \pm \\
17.5\end{array}$ & $\begin{array}{c}140.9 \pm \\
12.7\end{array}$ & $\begin{array}{c}114.9 \pm \\
10.5\end{array}$ & $\begin{array}{c}91.57 \pm \\
5.27\end{array}$ & $\begin{array}{c}121.3 \pm \\
11.8\end{array}$ & $\begin{array}{c}144.2 \pm \\
15.9\end{array}$ & $\begin{array}{c}188.4 \pm \\
13.5\end{array}$ & $\begin{array}{c}171.1 \pm \\
21.4\end{array}$ & $\begin{array}{c}45.22 \pm \\
6.36\end{array}$ \\
\hline 70 & 957 & $\begin{array}{l}100- \\
52-7\end{array}$ & benzaldehyde & P3 & $\begin{array}{c}18.77 \pm \\
2.55\end{array}$ & $\begin{array}{c}7.87 \pm \\
1.21\end{array}$ & nd & $\begin{array}{c}19.84 \pm \\
1.19\end{array}$ & $\begin{array}{c}36.15 \pm \\
2.08\end{array}$ & nd & $\begin{array}{c}29.33 \pm \\
3.02\end{array}$ & nd & $\begin{array}{c}4.22 \pm \\
0.26\end{array}$ \\
\hline 71 & 1042 & $\begin{array}{l}122- \\
78-1\end{array}$ & benzeneacetaldehyde & P4 & $\begin{array}{c}112.8 \pm \\
9.57\end{array}$ & $\begin{array}{c}13.03 \pm \\
2.12\end{array}$ & nd & $\begin{array}{c}51.34 \pm \\
4.17\end{array}$ & $\begin{array}{c}201.6 \pm \\
16.2\end{array}$ & nd & $\begin{array}{c}117.9 \pm \\
19.8\end{array}$ & nd & nd \\
\hline 72 & 1115 & $\begin{array}{l}124- \\
19-6\end{array}$ & nonanal & $P 5^{\mathrm{e}}$ & nd & nd & nd & nd & 0 & nd & nd & nd & $\begin{array}{c}25.95 \pm \\
3.65\end{array}$ \\
\hline 73 & 1720 & $\begin{array}{c}2765- \\
11-9\end{array}$ & pentadecanal & $\mathrm{P}^{\mathrm{e}}$ & nd & nd & nd & nd & $\begin{array}{c}4.06 \pm \\
0.54\end{array}$ & $\begin{array}{c}19.02 \pm \\
2.11\end{array}$ & $\begin{array}{c}10.14 \pm \\
0.84\end{array}$ & $\begin{array}{c}26.77 \pm \\
3.24\end{array}$ & nd \\
\hline 74 & 1307 & $\begin{array}{l}112- \\
44-7\end{array}$ & undecanal & P7 & $\begin{array}{c}1.99 \pm \\
0.21\end{array}$ & $\begin{array}{c}1.93 \pm \\
0.17\end{array}$ & nd & nd & nd & nd & nd & nd & $\begin{array}{c}26.82 \pm \\
3.96\end{array}$ \\
\hline 75 & 1262 & $\begin{array}{c}5392- \\
40-5\end{array}$ & 3,7-dimethyl-2,6-octadienal & $P 8^{\mathrm{e}}$ & $\begin{array}{c}6.16 \pm \\
0.81\end{array}$ & $\begin{array}{c}1.64 \pm \\
0.28\end{array}$ & nd & nd & nd & nd & nd & nd & $\begin{array}{c}557.4 \pm \\
69.7\end{array}$ \\
\hline 76 & 1833 & $\begin{array}{l}629- \\
80-1\end{array}$ & hexadecanal & $P 9^{e}$ & nd & nd & nd & nd & nd & nd & nd & nd & $\begin{array}{c}16.02 \pm \\
2.13\end{array}$ \\
\hline 77 & 870 & $\begin{array}{l}111- \\
27-3\end{array}$ & 1-hexanol & $\mathrm{P} 10$ & nd & nd & nd & nd & $\begin{array}{c}11.57 \pm \\
1.26\end{array}$ & nd & nd & nd & $\begin{array}{c}31.91 \pm \\
4.17\end{array}$ \\
\hline 78 & 872 & $\begin{array}{l}928- \\
95-0\end{array}$ & trans-2-hexenol & P11 & $\begin{array}{c}15.75 \pm \\
1.36\end{array}$ & nd & $\begin{array}{c}20.73 \pm \\
1.87\end{array}$ & nd & nd & $\begin{array}{c}38.91 \pm \\
4.56\end{array}$ & nd & $\begin{array}{c}22.89 \pm \\
3.23\end{array}$ & nd \\
\hline & & & Total & & $\begin{array}{c}471.1 \pm \\
27.5\end{array}$ & $\begin{array}{c}225.8 \pm \\
32\end{array}$ & $\begin{array}{c}177.9 \pm \\
16\end{array}$ & $\begin{array}{c}260.3 \pm \\
18.3\end{array}$ & $\begin{array}{c}471.4 \pm \\
34\end{array}$ & $\begin{array}{c}231.4 \pm \\
15.4\end{array}$ & $\begin{array}{c}447.3 \pm \\
29.6\end{array}$ & $\begin{array}{c}281.5 \pm \\
16.5\end{array}$ & $\begin{array}{c}799.2 \pm \\
55.3\end{array}$ \\
\hline & & & Relatives (\%) & & $7.36 \%$ & $2.85 \%$ & $2.80 \%$ & $4.89 \%$ & $4.39 \%$ & $3.59 \%$ & $10.53 \%$ & $2.28 \%$ & $7.00 \%$ \\
\hline & & & & & & Esters & & & & & & & \\
\hline 79 & 1263 & $\begin{array}{l}115- \\
95-7\end{array}$ & linalyl acetate & E1 & nd & nd & $\begin{array}{c}931.2 \pm \\
86.5\end{array}$ & nd & nd & nd & nd & nd & nd \\
\hline 80 & 1341 & $\begin{array}{l}134- \\
20-3\end{array}$ & methyl anthranilate & E2 & $\begin{array}{c}98.18 \pm \\
6.37\end{array}$ & nd & $\begin{array}{c}22.97 \pm \\
3.18\end{array}$ & $\begin{array}{c}4.76 \pm \\
0.57\end{array}$ & $\begin{array}{c}12.12 \pm \\
1.68\end{array}$ & $\begin{array}{c}157.5 \pm \\
13.4\end{array}$ & $\begin{array}{c}5.48 \pm \\
0.39\end{array}$ & $\begin{array}{c}49.47 \pm \\
3.38\end{array}$ & nd \\
\hline 81 & 1366 & $\begin{array}{l}141- \\
12-8\end{array}$ & neryl acetate & E3 & nd & nd & $\begin{array}{c}24.81 \pm \\
3.25\end{array}$ & nd & nd & nd & nd & $\begin{array}{c}8.69 \pm \\
0.64\end{array}$ & nd \\
\hline 82 & 1385 & $\begin{array}{l}105- \\
87-3\end{array}$ & geraniol acetate & $\mathrm{E} 4$ & nd & nd & $\begin{array}{c}47.43 \pm \\
5.11\end{array}$ & nd & nd & $\begin{array}{c}1.02 \pm \\
0.05\end{array}$ & nd & $\begin{array}{c}5.23 \pm \\
0.32\end{array}$ & nd \\
\hline & & & Total & & $\begin{array}{c}98.2 \pm \\
5.64\end{array}$ & nd & $\begin{array}{c}1026 \pm \\
92.1\end{array}$ & $4.8 \pm 0.27$ & $\begin{array}{c}12.1 \pm \\
1.36\end{array}$ & $\begin{array}{c}158.5 \pm \\
13.6\end{array}$ & $5.5 \pm 0.29$ & $\begin{array}{c}63.4 \pm \\
0.46\end{array}$ & nd \\
\hline & & & Relatives (\%) & & $1.53 \%$ & $0.00 \%$ & $16.17 \%$ & $0.09 \%$ & $0.11 \%$ & $2.46 \%$ & $0.13 \%$ & $0.51 \%$ & $0.00 \%$ \\
\hline & & & & & & Others & & & & & & & \\
\hline 83 & 1398 & $\begin{array}{l}488- \\
10-8\end{array}$ & cis-jasmone & $\mathrm{O} 1$ & $\begin{array}{c}8.85 \pm \\
0.65\end{array}$ & $\begin{array}{c}12.64 \pm \\
1.33\end{array}$ & $\begin{array}{c}2.44 \pm \\
0.27\end{array}$ & $\begin{array}{c}9.35 \pm \\
0.86\end{array}$ & $\begin{array}{c}27.22 \pm \\
3.45\end{array}$ & nd & nd & $\begin{array}{c}0.49 \pm \\
0.05\end{array}$ & nd \\
\hline 84 & 1081 & $\begin{array}{l}100- \\
64-1\end{array}$ & cyclohexanone oxime & $\mathrm{O} 2^{\mathrm{e}}$ & $\begin{array}{c}5.54 \pm \\
0.58\end{array}$ & nd & nd & $\begin{array}{c}3.96 \pm \\
0.53\end{array}$ & $\begin{array}{c}11.94 \pm \\
1.98\end{array}$ & nd & $\begin{array}{c}3.45 \pm \\
0.42\end{array}$ & nd & nd \\
\hline 85 & 1089 & $\begin{array}{c}1195- \\
32-0\end{array}$ & p-dimethylstyrene & $\mathrm{O} 3$ & nd & $\begin{array}{c}518.6 \pm \\
29.6\end{array}$ & nd & $\begin{array}{c}170.3 \pm \\
15.3\end{array}$ & $\begin{array}{c}348.6 \pm \\
45.8\end{array}$ & nd & nd & nd & nd \\
\hline 86 & 1138 & $\begin{array}{c}6909- \\
30-4\end{array}$ & trans- limonene oxide & $\mathrm{O} 4^{\mathrm{e}}$ & nd & nd & nd & nd & nd & $\begin{array}{c}33.25 \pm \\
37.6\end{array}$ & nd & $\begin{array}{c}118.4 \pm \\
22.1\end{array}$ & $\begin{array}{c}22.52 \pm \\
3.67\end{array}$ \\
\hline 87 & 1140 & $\begin{array}{l}140- \\
29-4\end{array}$ & benzyl nitrile & $\mathrm{O} 5$ & $\begin{array}{c}32.06 \pm \\
4.54\end{array}$ & nd & nd & $\begin{array}{c}48.99 \pm \\
3.65\end{array}$ & $\begin{array}{c}71.15 \pm \\
3.97\end{array}$ & nd & $\begin{array}{c}29.83 \pm \\
4.12\end{array}$ & nd & nd \\
\hline
\end{tabular}




\begin{tabular}{|c|c|c|c|c|c|c|c|c|c|c|c|c|c|}
\hline 88 & 1141 & $\begin{array}{c}57396- \\
75-5\end{array}$ & $\begin{array}{l}\text { 3,4-dimethyl-2,4,6- } \\
\text { octatriene }\end{array}$ & $\mathrm{O}^{\mathrm{e}}$ & $\begin{array}{c}7.89 \pm \\
1.05\end{array}$ & $\begin{array}{c}11.37 \pm \\
1.56\end{array}$ & $\begin{array}{c}31.59 \pm \\
4.37\end{array}$ & $\begin{array}{c}13.03 \pm \\
2.08\end{array}$ & $\begin{array}{c}13.38 \pm \\
1.56\end{array}$ & $\begin{array}{c}18.69 \pm \\
2.03\end{array}$ & $\begin{array}{c}4.27 \pm \\
0.34\end{array}$ & $\begin{array}{c}12.89 \pm \\
0.85\end{array}$ & nd \\
\hline 89 & 1292 & $\begin{array}{l}120- \\
72-9\end{array}$ & indole & O7 & $\begin{array}{c}42.83 \pm \\
6.37\end{array}$ & nd & $\begin{array}{c}1.33 \pm \\
0.31\end{array}$ & nd & $\begin{array}{c}26.45 \pm \\
1.54\end{array}$ & $\begin{array}{c}38.83 \pm \\
3.24\end{array}$ & $\begin{array}{c}25.02 \pm \\
1.59\end{array}$ & $\begin{array}{c}53.74 \pm \\
3.88\end{array}$ & nd \\
\hline 90 & 1500 & $\begin{array}{l}629- \\
62-9\end{array}$ & pentadecane & O8 & nd & nd & nd & $\begin{array}{c}14.26 \pm \\
1.28\end{array}$ & nd & nd & nd & $\begin{array}{c}5.44 \pm \\
0.61\end{array}$ & $\begin{array}{c}95.76 \pm \\
5.65\end{array}$ \\
\hline 91 & 1581 & $\begin{array}{c}1139- \\
30-6\end{array}$ & caryophyllene oxide & O9 & nd & nd & $\begin{array}{c}15.25 \pm \\
0.56\end{array}$ & nd & nd & nd & nd & nd & $\begin{array}{c}9.68 \pm \\
1.05\end{array}$ \\
\hline 92 & 1677 & $\begin{array}{c}2579- \\
04-6\end{array}$ & 8-heptadecene & $\mathrm{O} 10^{\mathrm{e}}$ & $\begin{array}{c}34.05 \pm \\
5.23\end{array}$ & nd & $\begin{array}{c}9.47 \pm \\
1.21\end{array}$ & $\begin{array}{c}64.43 \pm \\
2.37\end{array}$ & nd & nd & $\begin{array}{c}14.14 \pm \\
2.18\end{array}$ & nd & $\begin{array}{c}124.7 \pm \\
15.8\end{array}$ \\
\hline 93 & 1678 & $\begin{array}{c}6765- \\
39-5\end{array}$ & 1-heptadecene & $\mathrm{O} 11^{\mathrm{e}}$ & nd & $\begin{array}{c}8.29 \pm \\
0.67\end{array}$ & nd & nd & $\begin{array}{c}24.42 \pm \\
3.35\end{array}$ & nd & nd & nd & nd \\
\hline \multirow[t]{7}{*}{94} & \multirow[t]{7}{*}{1703} & $\begin{array}{l}629- \\
78-7\end{array}$ & heptadecane & $\mathrm{O} 12$ & $\begin{array}{c}1.04 \pm \\
0.02\end{array}$ & nd & nd & $\begin{array}{c}10.05 \pm \\
1.27\end{array}$ & $\begin{array}{c}4.22 \pm \\
0.53\end{array}$ & nd & nd & nd & nd \\
\hline & & & Total & & $\begin{array}{c}132.3 \pm \\
9.65\end{array}$ & $\begin{array}{c}550.9 \pm \\
39.4\end{array}$ & $\begin{array}{c}60.08 \pm \\
3.87\end{array}$ & $\begin{array}{c}334.1 \pm \\
19.7\end{array}$ & $\begin{array}{c}527.4 \pm \\
54.2\end{array}$ & $\begin{array}{c}90.77 \pm \\
6.38\end{array}$ & $\begin{array}{c}76.71 \pm \\
5.32\end{array}$ & $\begin{array}{c}190.5 \pm \\
15.6\end{array}$ & $\begin{array}{c}252.7 \pm \\
32.1\end{array}$ \\
\hline & & & Relatives(\%) & & $2.07 \%$ & $6.95 \%$ & $0.95 \%$ & $6.28 \%$ & $4.91 \%$ & $1.41 \%$ & $1.81 \%$ & $1.54 \%$ & $2.21 \%$ \\
\hline & & & Terpene & & $\begin{array}{c}5698 \pm \\
319\end{array}$ & $\begin{array}{c}7155 \pm \\
299\end{array}$ & $\begin{array}{c}5083 \pm \\
286\end{array}$ & $\begin{array}{c}4721 \pm \\
321\end{array}$ & $\begin{array}{c}9729 \pm \\
452\end{array}$ & $\begin{array}{c}5959 \pm \\
225\end{array}$ & $\begin{array}{c}3716 \pm \\
187\end{array}$ & $\begin{array}{c}11818 \pm \\
658\end{array}$ & $\begin{array}{c}10366 \pm \\
489\end{array}$ \\
\hline & & & Telatives(\%) & & $89.04 \%$ & $90.21 \%$ & $80.08 \%$ & $88.74 \%$ & $90.59 \%$ & $92.54 \%$ & $87.53 \%$ & $95.67 \%$ & $90.79 \%$ \\
\hline & & & Total & & $\begin{array}{c}6400 \pm \\
201\end{array}$ & $\begin{array}{c}7932 \pm \\
285\end{array}$ & $\begin{array}{c}6347 \pm \\
336\end{array}$ & $\begin{array}{c}5321 \pm \\
568\end{array}$ & $\begin{array}{c}10740 \pm \\
568\end{array}$ & $\begin{array}{c}6440 \pm \\
325\end{array}$ & $\begin{array}{c}4246 \pm \\
187\end{array}$ & $\begin{array}{c}12354 \pm \\
526\end{array}$ & $\begin{array}{c}11417 \pm \\
389\end{array}$ \\
\hline & & & Sorts & & 54 & 50 & 46 & 50 & 56 & 51 & 48 & 55 & 47 \\
\hline
\end{tabular}

${ }^{a}$ RI: retention indices were determined using a series of alkanes C5-C30 as external references.

${ }^{\mathrm{b}}$ CAS: Chemical abstracts service.

${ }^{\mathrm{c}}$ Code :M, monoterpenes; S, sesqueterpenes; H, alcohols; P, aliphatic adehydes; A, aldehydes; E, esters; O, others.

${ }^{\mathrm{d}}$ The content of blossom volatiles ( $\mu \mathrm{g} . \mathrm{g}^{-1} \mathrm{FW}$ equivalent of cyclohexanone).

e Compound reported in the present study for the first time.

${ }^{\mathrm{f}}$ Data are expressed as means \pm standard deviation of triplicate samples.

${ }^{\mathrm{g}}$ Numbers in bold present the dominant compounds for each species.

Identification of volatile compounds was first based on their retention indices (RI) relative to (C5-C20) n-alkane with those of the authentic compounds in the database. They were further confirmed by comparing mass spectral fragmentation patterns with data libraries (NIST-98 and FLAVOUR 2.0) of GC-MS data systems and other published mass spectra. The relative content of each compound was calculated by using the peak areas of all of the compounds relative to an internal standard (cyclohexanone).

\subsection{Data Analysis}

All data are expressed as means \pm standard deviation of three replicates. The mean values were used for further analysis. Principal component analysis (PCA) and hierarchical cluster analysis (HCA) were performed using SPSS v19.0 software (SPSS Inc., Chicago, IL, USA). OriginPro 7.5 G (Microcal Software, Inc., Northampton, MA, USA) was used to determine the standard deviation and the variation patterns.

\section{Results and Discussion}

\subsection{Yields and Citrus Flower Volatile Components}

The yield of flower volatile components in the Citrus species/varieties studied varied from 4249 to $12354 \mu \mathrm{g} \mathrm{g}-1$ FW. The highest yield was found in Ningmeng 4, and the lowest in Changshanhuyou (Table 2 and Figure 1). Significantly higher total volatile contents were found in Ningmeng 4, Dannaxiangyuan and Taiwanpenggan than in other genotypes. From the citrus flowers analyzed in this study, a total of 149 compounds were detected, 94 of

which had volatile contents over $10 \mu \mathrm{g} \mathrm{g}^{-1} \mathrm{FW}$, while others had trace amounts. The 94 compounds included 20 monoterpenes, 27 sesquiterpenes, 12 terpene alcohols, 5 terpene aldehydes, 11 aliphatic aldehydes and alcohols, 4 esters and 12 others; 31 of these compounds are reported for the first time in this study (Table 2). Monoterpenes (38.04-82.59\%), sesquiterpenes (6.70-26.42\%), terpene alcohols (0.59-43.25\%) and terpene aldehydes (0.02$6.50 \%)$ were the major components of citrus flower volatiles (Figure 1, Table 2) and accounted for up to $92.4 \%$ of the total volatiles.

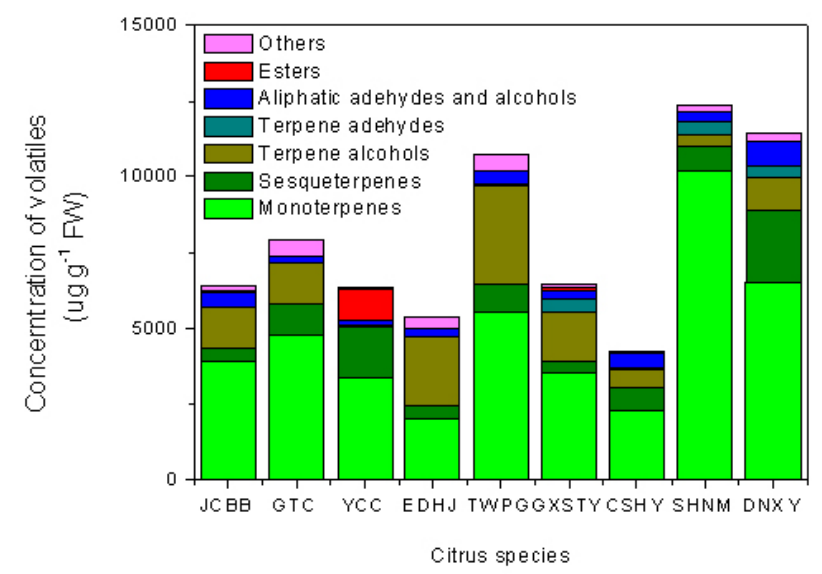

Figure 1. The main volatile components identified in the flowers of nine citrus genotypes studied in the present study and their contents variation

Monoterpenes were the most predominant compounds in all of the species/varieties analyzed. The highest level of monoterpenes was found in Ningmeng $4\left(10203 \mu \mathrm{g} \mathrm{g}^{-1}\right.$ $\mathrm{FW}$ ), and the lowest in Edanhongju (2024 $\left.\mu \mathrm{g} \mathrm{g}^{-1} \mathrm{FW}\right)$. Limonene was the most abundant monoterpene (60.91$5650 \mu \mathrm{g} \mathrm{g}^{-1} \mathrm{FW}$ ), followed by $\gamma$-terpinene. The highest level of limonene was found in Ningmeng 4, and the 
lowest was found in Yichangcheng. Limonene contributed to almost $45.7 \%$ and $45.8 \%$ of the total monoterpenes in Ningmeng 4 and Dannaxiangyuan, respectively, and making the two varieties distinct from all of the other varieties studied. Limonene, $\alpha$-terpinene, $\gamma$-terpinene and neo-alloocimene were detected in all of the species/varieties tested. (-)- $\alpha$-pinene was only detected in Jinchengbeibei 447 and Dannaxaingyuan, and (-)- $\beta$-pinene was only in Edanhongju and Dannaxaingyuan. Cosmene was only in Yichangcheng and Guangxishatianyou. High levels of $\gamma$-terpinene were detected in Goutoucheng, Taiwanpenggan and Changshanhuyou (> $848 \mu \mathrm{g} \mathrm{g}^{-1} \mathrm{FW}$ ). A high level of (e)-ocimene was detected in Guangxishatianyou (1172.61 $\left.\mathrm{g} \mathrm{g} \mathrm{g}^{-1} \mathrm{FW}\right)$ and Ningmeng 4 $\left(1173 \mu \mathrm{g} \mathrm{g}^{-1} \mathrm{FW}\right)$. High levels of $\beta$-pinene were detected in Guangxishatianyou (954.2 $\left.\mu \mathrm{g} \mathrm{g}^{-1} \mathrm{FW}\right)$ and Ningmeng 4 $\left(1629.3 \mu \mathrm{g} \mathrm{g}^{-1} \mathrm{FW}\right)$. High levels of sabinene were detected in Jinchengbeibei $447 \quad\left(1610 \quad \mu g \quad g^{-1} \quad F W\right)$ and Taiwanpenggan (1811 $\left.\mu \mathrm{g} \mathrm{g}^{-1} \mathrm{FW}\right)$ (Table 2).

Sesquiterpenes were the second most abundant components (375.8-2369 $\mu \mathrm{g} \mathrm{g}^{-1} \mathrm{FW}$ ) of the volatile compounds analyzed. The highest levels of sesquiterpenes were found in Dannaxaingyuan, and the lowest in Guangxishatianyou. Caryophyllene (69.95-897.8 $\mu \mathrm{g} \mathrm{g}^{-1}$ FW) was the main component of the sesquiterpenes tested, followed by $\beta$-elemene and $\alpha$-caryophyllene. The highest level of caryophyllene was found in Dannaxaingyuan, and the lowest in Edanhongju. Caryophyllene, $\alpha$-cubebene and $\alpha$-caryophyllene were present in all of the species/varieties examined. $\beta$-bourbonene, trans- $\alpha$-bergamotene and $\delta$ guaiene were only detected in Yichangcheng; $\beta$ sesquiphellandrene was only in Taiwanpenggan; $\alpha$ guaiene was only in Yichangcheng and Changshanhuyou; and (+)-epi-bicyclosesquiphellandrene was only in Yichangcheng and Guangxishatianyou. High levels of copaene $\left(229.7 \mu \mathrm{g} \mathrm{g}^{-1} \mathrm{FW}\right)$ and L- $\beta$-bisabolene $(193.3 \mu \mathrm{g}$ $\left.\mathrm{g}^{-1} \mathrm{FW}\right)$ were detected in Yichangcheng. High levels of (z)- $\beta$-farnesene were detected in Goutoucheng $(253.3 \mu \mathrm{g}$ $\left.\mathrm{g}^{-1} \mathrm{FW}\right)$ and Taiwanpenggan $\left(356.5 \mu \mathrm{g} \mathrm{g}^{-1} \mathrm{FW}\right)$.

Terpene alcohols were the third most abundant components (58.8-3267 $\mu \mathrm{g} \mathrm{g}^{-1} \mathrm{FW}$ ) in the volatile samples analyzed. The highest level of total terpene alcohol was found in Taiwanpenggan, and the lowest in Yichangcheng. Linalool (34.65-2099 $\left.\mu \mathrm{g} \mathrm{g}^{-1} \mathrm{FW}\right)$ was the main terpene alcohol of the tested citrus flowers, followed by $\alpha$ terpineol and geraniol. The highest level of linalool was found in Taiwanpenggan, composing $85.5 \%$ of the total alcohol; the lowest level was found in Yichangcheng. Linalool and $\alpha$-terpineol were identified in all of the species/varieties tested. D-nerolidol was only detected in Changshanhuyou and Dannaxaingyuan, and 2,3-dihydro6-trans-farnesol was only in Ningmeng 4 and Dannaxaingyuan; (e,e)-farnesol was only in Ningmeng 4. High levels of nerol (450.4 $\left.\mathrm{g} \mathrm{g} \mathrm{g}^{-1} \mathrm{FW}\right)$ and geraniol (345.3 $\left.\mu \mathrm{g} \mathrm{g}^{-1} \mathrm{FW}\right)$ were detected in Dannaxaingyuan. A high level of nerolidol was detected in Guangxishatianyou $\left(195.2 \mu \mathrm{g} \mathrm{g}^{-1} \mathrm{FW}\right)$. In this study, only 5 terpene aldehydes were detected in the varieties studied, and citronellal and neral were the major terpene aldehydes. l-perillaldehyde and 1,3,6,10-farnesatetraen-12-al were only detected in Jingchengbeibei 447 and Taiwanpenggan, while geranial was only detected in Yichangcheng and Taiwanpenggan.

Only 4 terpene aldehydes were identified in the citrus flowers of all of the species/varieties analyzed. Except for
Jinchengbeibei 447 and Goutoucheng, citronellal was detected in the flowers of all tested species/varieties. 1perillaldehyde and 1,3,6,10-farnesatetraen-12-al was only detected in Jinchengbeibei 447 and Taiwanpenggan. High levels of neral (349.9 $\mu \mathrm{g} \mathrm{g} \mathrm{g}^{-1}$ FW) were found in Dannaxiangyuan, and high levels of geranial in Guangxishatianyou (220.2 $\left.\mu \mathrm{g} \mathrm{g}^{-1} \mathrm{FW}\right)$ and Ningmeng 4 (233.6 $\left.\mu \mathrm{g} \mathrm{g}^{-1} \mathrm{FW}\right)$.

In this study, 11 aliphatic aldehydes and alcohols were detected in the species/varieties studied. Aliphatic aldehydes, such as hexanal and trans-2-hexenal, were the main aldehydes in citrus flowers and were detected in all of the citrus species/variety tested. High levels of 3,7dimethyl-2,6-octadienal were detected in Dannaxiangyuan (557.4 $\left.\mu \mathrm{g} \mathrm{g}^{-1} \mathrm{FW}\right)$. Only two alcohols were detected in these tested cultivars. 1-hexanol was only detected in Taiwanpenggan and Dannaxaingyuan, and trans-2hexenol was only detected in Jinchengbeibei 447, Yichangcheng, Guangxishatianyou and Ningmeng 4.

Esters were minor components in the volatile samples analyzed and varied significantly depending on the species/varieties (nd to $1026 \mu \mathrm{g} \mathrm{g}^{-1} \mathrm{FW}$ ). The highest level of esters was found in Yichangcheng. Methyl anthranilate was the predominant ester in all of the flowers tested. The highest level was found in Taiwanpenggan, and a relatively high level was also found in Yichangcheng (16.17\%). All four esters, and an especially high level of linalyl acetate $\left(931.2 \mu \mathrm{g} \mathrm{g}^{-1} \mathrm{FW}\right)$, were detected in Yichangcheng, but no esters were found in Dannaxiangyuan. In addition, alkenes, indoles, and terpene oxides were found in the citrus volatile samples. Especially high levels of p-dimethylstyrene were detected in Goutoucheng $\left(518.6 \mu \mathrm{g} \mathrm{g}^{-1} \mathrm{FW}\right)$ and Taiwanpenggan (348.6 $\left.\mu \mathrm{g} \mathrm{g}^{-1} \mathrm{FW}\right)$.

In general, different genotypes were found to have different volatile components. In this study, (e)-ocimene, linalool and $\beta$-pinene were found to be the major volatile compounds in the Guangxishatianyou flower, and $\gamma$ terpinene and linolool were found to be the major volatile compounds in the Changshanhuyou flower. These results suggest that C. grandis flowers are abundant in linolool, but other characterized monoterpenes may depend on cultivars. The Yichangcheng flower was characterized by B-ocimene, linalyl acetate, caryophyllene and myrcene. Ningmeng 4 was rich in limonene, $\beta$-pinene and (e)ocimene, Dannaxiangyuan was found rich in limonene, caryophyllene and L- $\beta$-bisabolene.

It has also been well documented that different citrus species have their own characteristic volatiles. Attaway et al. (1966) reported $C$. sinensis was dominated by $\beta$ myrcene, linalool and $\alpha$-myrcene, but $\beta$-myrcene was the richest compound in $C$. reticulata. In addition, rich sabinene, myrcene, $(+)$-limonene, $\beta$-ocimene and linalool were found in petal and pistil oils of $C$. sinensis and $C$. reticulate. In the present study, we found that Jingchengbeibei 447 had high levels of sabinene (25.14\%), linalool (17.13\%) and limonene (15.86\%). Goutoucheng was abundant in $\gamma$-terpinene (20.03\%), linolool (15.07\%) and p-cymene (5.90\%). Edanhongju was abundant in linalool (38.21\%) and o-methylthymol (2.93\%). Taiwanpenggan was abundant in linalool (25.97\%) and $\beta$ ocimene $(12.12 \%)$. These results indicate that $C$. sinensis and $C$. reticulate flowers have similar major volatiles, but the volatile profiles are different from that of $C$. aurantium. 
Jabalpurwala et al. found that $C$. grandis flower volatiles were predominantly linalool, p-cymene and methyl anthranilate (Jabalpurwala et al. 2009), while Cheong et al. found that limonene, linalool, ethanol and cis- $\beta$-ocimene were the major volatiles for Malaysian pumelo flowers ( $C$. grandis Osbeck) (Cheong et al. 2011). Recently, Chung reported the volatiles of the Hallabong blossom (C. kiyomi $\times$ C. ponkan) (Chung 2012). They found that the most abundant volatile compounds of Hallabong were linalool, limonene, $\beta$-myrcene and sabinene. Numerous studies have shown that the flower oils of $C$. aurantium L. from different provinces primarily consist of linalool and limonene, although their percentages are different (Ramadan et al. 1996; Sarrou et al. 2013; Wei et al. 2010). These studies indicate that the flower volatiles might primarily be related to their genetic background (Figure 1).

\subsection{Flower Volatile Compositions and Variation Patterns between Hybrid Citrus Species and Their Presumed Parents}

The secondary metabolites of plants might be good complementary evidence for plant taxonomic study (Sumner et al. 2007). In the present study, the flower volatile components of nine citrus genotypes cultivated under the same climatic and horticultural conditions were examined, giving us opportunity to better evaluate the influence of genetic and/or province factors on the components and content of citrus volatile compounds.

It is known that Citrus volatile components are closely related to the genetic origin of the species/variety studied (Liu et al. 2013; González-Mas et al. 2011; Vekiari et al. 2001). To analyze the influence of genetic factors on the composition and content of citrus volatiles, the variation patterns of flower volatile components between the basic Citrus types in Swingle's system, i.e., Papeda ( $C$. ichangensis Swingle), Cephalocitrus (C. grandis (L.) Osbeck), Citrophorum (C. medica L.) and Sinocitrus ( $C$. reticulata Blanco), and their presumed hybrids, including Citrus sinensis (L) Osbeck, C. aurantium L., C. paradis Macf., and C. limon (L) Burm.f. were investigated. There were clear differences in volatile components between four basic Citrus types (Figure 2). As shown in Figure 2A and Table 1, Papeda contained the highest amounts of $\beta$ ocimene, linalyl acetate and myrcene. Sinocitrus had the highest levels of linalool and cavocrol. No characteristic volatile compounds were detected in Cephalocitrus, but this Citrus type has more $\beta$-pinene and limonene than Papeda and Sinocitrus. Citrophorum had the highest level of limonene, and high levels of $\beta$-pinene, caryophyllene and L- $\beta$-bisabolene were also detected in Citrophorum. The presence of 3,7-dimethyl-2,6-octadienal was unique to Citrophorum. These results are consistent with previous reports (Flamini et al. 2007; Hosni et al. 2010), indicating that different Citrus types are characterized by unique variation patterns in the composition and content of volatile compounds. In addition, our data suggests that Papeda, Citrophorum and Sinocitrus may be excellent sources of linalyl acetate, limonene and linalool, respectively.

In the Citrus genus, sweet orange, sour orange, grapefruit and lemon are well-accepted hybrids. To analyze the influence of hybridization on the flower volatile components, in this study we perform a detailed comparison of the variation patterns of the volatile compounds of these hybrids with those of their presumed parents.

It is well known that sweet orange is a hybrid between pumelo (C. grandis L.) and mandarin (C. reticulate) (Li et al. 2010). The differences in the composition and content of 94 volatile compounds identified in this study from Citrus sinensis Osbeck cv. Jinchengbeibei 447, C. grandis Osbeck cv. Guangxishatianyou and C. reticulata Blanco cv. Edanhongju are shown in Figure 2B. There were 24 volatiles widely distributed in sweet orange and its presumed parents. Unlike its presumed parents, the sweet orange contained significantly higher levels of myrcene, (z)-ocimene, $\beta$-elemene, $\alpha$-terpineol and trans-2-hexenal. Additionally, sabinene, (-)- $\alpha$-pinene, $\alpha$-phellandrene, 3 carene, and (-)- $\alpha$-selinene were detected only in sweet orange.

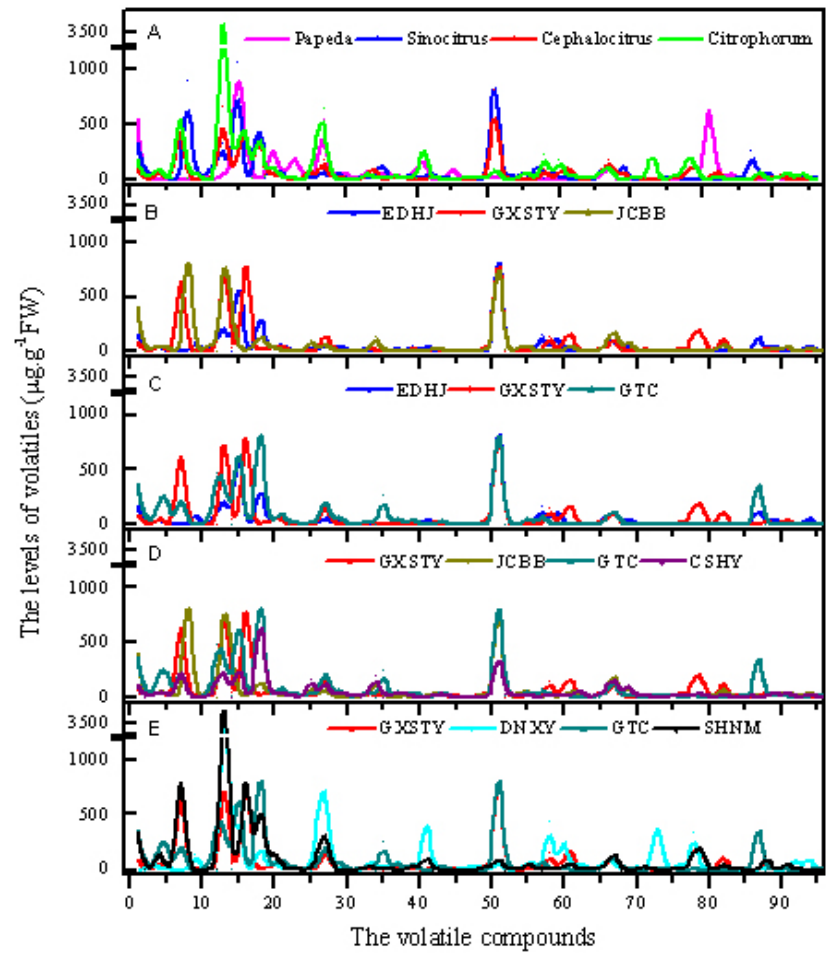

Figure 2. Variation patterns of the flower volatile compounds in basic Citrus species and their presumed hybrids

(A) variation patterns of four basic species-types; (B, C, D, E) variation patterns of the four hybrids (JCBB, GTC, CSHY, SHNM) and their presumed parents, respectively.

Most researchers suggest that sour orange is a hybrid of pumelo and mandarin ( $\mathrm{Li}$ et al. 2010; Nicolosi et al. 2000). Figure 2C shows differences on the composition between C. aurantium L. cv. Goutoucheng and C. grandis Osbeck cv. Guangxishatianyou and $C$. reticulata Blanco cv. Edanhongju and the content of the 94 volatile compounds identified in this study. Twenty-six common volatiles were found in sour orange and its presumed parents pumelo and mandarin. Of the components detected above, the level of 13 volatiles, including $\gamma$-terpinene, elemene, $\alpha$-cubebene, $\beta$-elemene, caryophyllene, $\beta$-cubebene, $\gamma$ elemene, $\alpha$-caryophyllene, germacrene $\mathrm{D}$, elixene, $\delta$ cadinene, $\alpha$-terpineol and copaene were significantly higher in sour orange than the presumed parents, especially $\alpha$-terpinene and caryophyllene. The levels of $\alpha$ caryophyllene, $\delta$-cadinene, p-cymene, p-dimethylstyrene, $(\mathrm{z})-\beta$-farnesene and $\mathrm{p}$-dimethylstyrene in sour orange were 
significantly higher than in their presumed parents. $\alpha$ phellandrene, $\alpha$-bergamotene, L-alloaromadendrene, phenethyl alcohol, (-)-4-terpineol, cis-jasmone and 1heptadecene were detected only in the hybrids. The sour orange also had a higher content of common volatiles than its presumed parents. Notably, the sour orange was abundant in many unique volatiles, which showed that sour orange has remarkable heterosis and great potential for future utilization. These results suggest that the variation pattern in Goutoucheng is more similar to Edanhongju than Guangxishatianyou.

Changshanhuyou is thought to be a hybrid of $C$. sinensis and C. grandis (Nicolosi et al. 2000). Figure 2D shows the composition and content differences of the 94 volatile compounds identified in this study between Changshanhuyou (C. paradisi cv. Changshanhuyou), Citrus sinensis Osbeck cv. Jinchengbeibei 447, and C. aurantium L. cv. Goutoucheng. There were 21 common volatiles in the hybrids and their presumed parents. The levels of $\beta$-pinene, $\gamma$-terpinene, $\beta$-elemene and hexanal in the hybrids were significantly higher than those in their presumed parents. D-nerolidol was found only in Changshanhuyou. These results suggest that the variation pattern of Changshanhuyou is very similar to its presumed parents, especially that of Goutoucheng. The contents of volatiles identified in Changshanhuyou were all lower than in its presumed parents.

Lemon is widely thought to be a hybrid of citron and sour orange (Nicolosi et al. 2000; Barrett et al. 1976; Lu et al. 2011; Malik et al. 1974) Figure 2E shows the composition and content differences in the 94 volatile compounds identified in this study between C. limon (L). Burm.f cv. Ningmeng 4, C. grandis Osbeck cv. Guangxishatianyou, C. medica L cv. Dannaxaingyuan and C. aurantium L. cv. Goutoucheng. The compounds $\alpha-$ terpinene, limonene, neo-alloocimene, $\alpha$-cubebene, caryophyllene, $\alpha$-caryophyllene, $\gamma$-muurolene, linalool, $\alpha$ terpineol, hexanal and trans-2-hexenal were all detected in lemon and its presumed parents. Significantly higher levels of limonene, $\beta$-pinene, (e)-ocimene, $\delta$-terpinene and pentadecanal were detected in lemon than in its presumed parents. The origin of lemon was thought to be divergent for a long time. Our research of the flower volatile variation patterns of lemon shows its proposed parents are closely related (Figure 2E); the variation pattern of the hybrid is exactly the same as its parents.

\subsection{The Taxonomic Implications of the Compositional Variation of Citrus Flower Volatile Compounds}

To test the taxonomic value of citrus flower volatiles, principal component analysis (PCA) and hierarchical cluster analysis (HCA) were carried out using the 94 compounds identified in this study. PCA analysis (Figure 3A, 3B) showed that the first two principal components accounted for $65.11 \%$ of the total variance and were high enough to represent all of the variables. Figure 3A showed the scatter plot of scores of principal components 1 and 2. Figure $3 \mathrm{~B}$ showed the corresponding loading plot. The Citrus species/varieties studied could be divided into two major groups (Groups I and II), with group II being further divided into three subgroups (Subgroups IIa, IIb and IIc) (Figure 3A). The four basic Citrus types were clearly separated in two groups and three subgroups based on the PCA results. Citrophorum includes Dannaxiangyuan and Ningmeng 4 and is clustered in group I, group I, which was characterized by a high terpene content. Sinocitrus included Edanhongju and Taiwanpenggan and was clustered in subgroup IIa, and Goutoucheng was grouped with Edanhongju. Cephalocitrus included Guangxishatianyou and Changshanhuyou and was in subgroup IIb. Papeda, which was characterized by esters, was in subgroup IIc. Regarding the hybrids, Goutoucheng and Jinchengbeibei 447 were grouped with Guangxishatianyou and Edanhongju. Sihaonignmeng was grouped with Dannaxiangyuan, Guangxishatianyou and Goutoucheng. So the four basic types of the genus Citrus L., Papeda, Cephalocitrus, Citrophorum and Sinocitrus, can be clearly separated based on the cluster analysis of their volatile compounds in the PCA plot. All of the presumed hybrid species, including Jinchengbeibei 447 ( $C$. sinensis Osb.), Goutoucheng (C. aurantium L.), Ningmeng 4 (C. limon Burm.f.), and Changshanhuyou (C. paradisi cv. Changshanhuyou), grouped closely together with one of their suggested parent species in the constructed dendrogram. These results revealed that the components of citrus flower volatiles were variable among different species/varieties types, although underlying intraspecific similarities and interspecific chemical varieties were found in flower volatiles. Similar findings have been reported for the chemical polymorphisms of citrus essential oils and leaf volatiles (Azam et al. 2013; Lota et al. 2000).
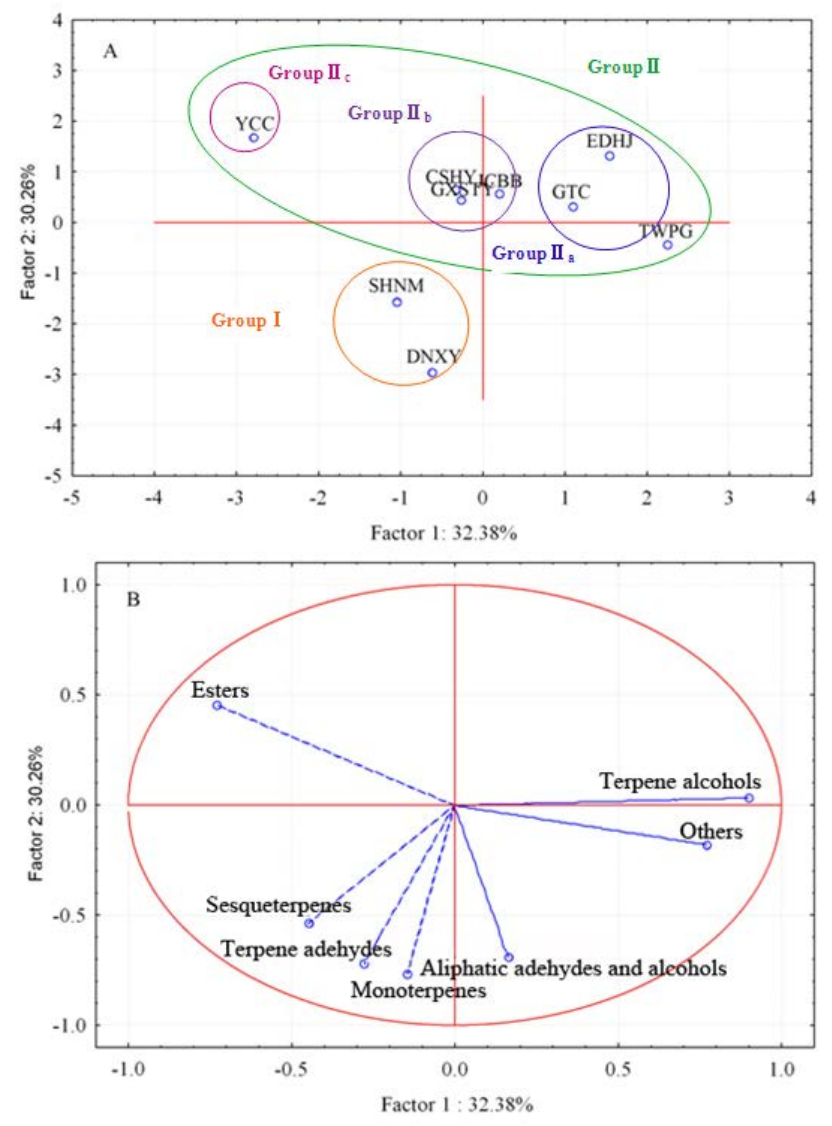

Figure 3. Principal component analysis (PCA) of Citrus flower volatiles:(A) score plot for the first and second principal components; and (B) loading plot of for the first and second principal components. Volatile variables explained according to the blossom volatile categories of the studied Citrus species 
HCA was also carried out to explore the classification value of the citrus volatile compounds (Figure $3 \mathrm{~B}$ ). The results are shown in Figure 4. Nine of the species/varieties studied were grouped into two main clusters. Cluster I consists of C. limon (L) Burm.f. and C. medica L. and was characterized by a high level of limonene. This was inconsistent with the results from PCA analysis. Cluster II included three subgroups (IIa, IIb, IIc). Cluster IIa consisted of C. aurantium, C. reticulata (Edanhongju) and C. paradisi cv. Changshanhuyou. In Cluster IIa, Goutoucheng was rich in $\gamma$-terpinene, and Edanhongju had a higher content of o-methylthymol, and Changshanhuyou was rich in $\gamma$-terpinene. Cluster IIb included C. sinensis and $C$. grandis, which were distinct from others because of their high methyl anthranilate content. Cluster IIc was composed of $C$. ichangensis and $C$. reticulata (Taiwanpenggan) and was characterized by high 3,7dimethyl-1,3,6-octatriene and myrcene content.

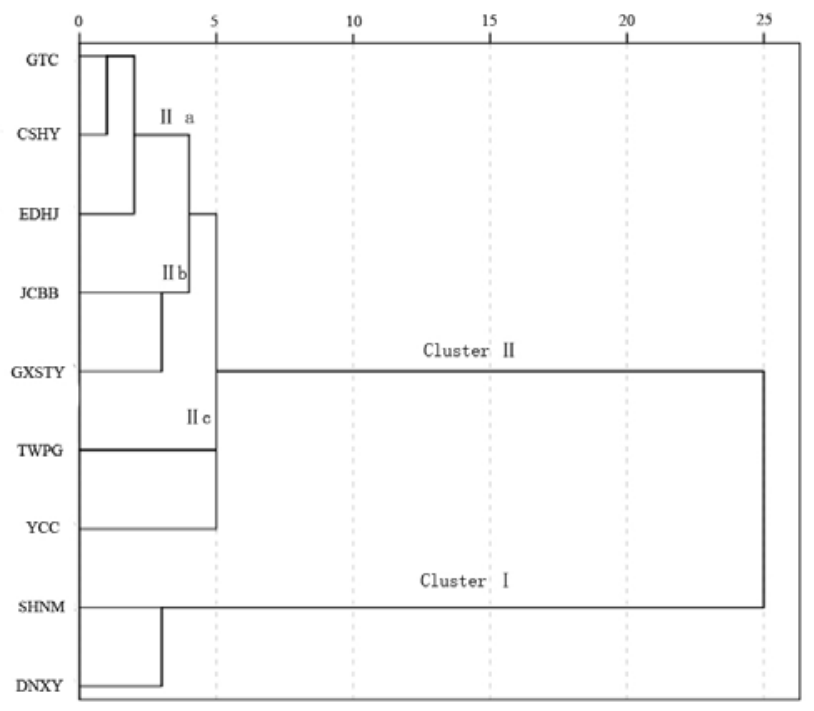

Figure 4. The dendrogram of the Citrus species studied based on the 94 flower volatile compounds (Table 1)

These results are in agreement with other molecular evidence from previous studies. Our previous studies demonstrated that sweet orange and sour orange were hybrids of mandarin (C. reticulata) and pumelo ( $C$. grandis) by using internal transcribed spacer (ITS) and chloroplast DNA sequences and amplified fragment length polymorphism (AFLP) fingerprints. In this study, though similar volatiles pattern were not observed in Jinchengbeibei 447 or in their presumed parents, the majority of volatiles identified were common. The PCA and HCA model showed that the hybrid and its presumed parents share the same flower volatile profile, which provides the chemotaxonomic evidence that is in agreement with previous results based on enzyme and molecular evidence ( $\mathrm{Li}$ et al. 2011; Barkley et al. 2006). Similar results were observed in Changshanhuyou. In this study, a very close variation pattern of flower volatiles was found in Ningmeng 4 and its proposed parents, which further confirmed the hypothesis that rough lemon is a cross between citron and mandarin (Miller et al. 2011; Rossi et al. 2013).

$\mathrm{Li}$ et al. analyzed the phylogenetic relationship of the four basic biotypes of Citrus, namely, Papeda, pumelo, citron and tangerine, using chloroplast DNA regions (cpDNA), amplified fragment length polymorphisms
(AFLP) fingerprints and nuclear internal transcribed spacer (ITS). The molecular data of their study indicated that the Citrus genus was of monophyletic origin ( $\mathrm{Li}$ et al. 2010), which is consistent with our current PCA and HCA results (Figure 3A). Their cpDNA results showed that $C$. grandis is closely related to the Papeda species. In our PCA plot, Changshanhuyou and Guangxishatianyou (in IIb) are close to Taiwanpenggan and Edanhongju (in IIc). The AFLP dendrogram shows that Ichang papeda, C. Reticulata and C. grandis are more closely related to each other than they are to other types in the genus and that papeda is distant from $C$ reticulate ( $\mathrm{Li}$ et al. 2010), which was consistent with our PCA and HCA results.

\section{Conclusion}

The composition and content variation of flower volatiles in seven native Chinese citrus species/varieties, namely, Jinchengbeibei 447, Goutoucheng, Yichangcheng, Edanhongju, Taiwanpenggan, Guangxishatianyou and Sihaoningmeng, are reported for the first time in this study. Monoterpenes (36.85-82.59\%), sesquiterpenes (5.83$26.42 \%)$ and terpene alcohols $(0.91-44.82 \%)$ were the major citrus flower volatile components. Monoterpenes were the most predominant compounds in all of the species/varieties analyzed, and limonene was the most abundant monoterpene followed by $\gamma$-terpinene. Different species/varieties flower had characteristic volatiles. Papeda was characterized by high levels of $\beta$-ocimene, linalyl acetate, myrcene and neo-alloocimene. Citrophorum (Ningmengsihao 4 and Dannaxiangyuan) had the highest levels of limonene, and $\alpha$-caryophyllene. Cephelocitrus had the highest level of limonene, $\beta$-pinene and linolool. Sinocitrus had the highest level of linolool. Linolool was also the characteristic terpene in $C$. sinensis (L.) Osbeck and C. aurantium L. C. sinensis (L.) Osbeck (Jinchengbeibei 447) had a high level of limonene, while C. aurantium L. (Goutoucheng) had the highest level of $\gamma$ terpinene. The compositional variation patterns of the basic citrus species indicated strong genetic influence on the volatile compounds. The hybrid species/variety shared largely similar patterns to their presumed parents, which may be useful for chemotaxonomy. Overall, our study provides useful information for future citrus germplasm evaluation and utilization.

\section{Acknowledgements}

This work was supported by the National Natural Science Foundation of China (No. 31171930), the Natural Science Foundation of Chongqing City (cstc2013jcyjA80012), the Fundamental Research Funds for the Central Universities (XDJK2014A014), the Scientific and Technological Achievements Transformation fund of Chongqing city (cstc2014jcsfnycgzhA80009), the National Key Technology R\&D Program (No. 2007BAD47B07) and China Agriculture Research System (No. CARS-27).

\section{References}

[1] Al-Ababneh, S., Karam, N., Shibli, R. Cryopreservation of sour orange (Citrus aurantium L.) shoot tips. In vitro Cellular \& Developmental Biology-Plant, 38, (6): 602-607. 2002. 
[2] Anwar, F., Naseer, R., Bhanger, M. I., Ashraf, S., Talpur, F., Aladedunye, F. Physico-chemical characteristics of citrus seeds and seed oils from pakistan. Journal of The American Oil Chemists Society, 85, (4): 321-330. 2008.

[3] Attaway, J. A.; Pieringer, A. P., Barabas, L. J. The origin of citrus flavor components-II.: Identification of volatile components from citrus blossoms. Phytochemistry, 1966, 5, (6), 1273-1279.

[4] Azam, M., Jiang, Q., Zhang, B., Xu, C., Chen, K. Citrus leaf volatiles as affected by developmental stage and genetic type. International Journal of Molecular Sciences, 14, (9): 17744-17766. 2013.

[5] Barkley, N., Roose, M., Krueger, R., Federici, C. Assessing genetic diversity and population structure in a citrus germplasm collection utilizing simple sequence repeat markers (SSRs). Theoretical and Applied Genetics, 112, (8): 1519-1531. 2006.

[6] Barrett, H.C., Rhodes, A.M. A numerical taxonomic study of affinity relationships in cultivated citrusand its close relatives. Systematic Botany, 1:105-136. 1976.

[7] Boussaada, O., Skoula, M., Kokkalou, E., Chemli, R. Chemical variability of flowers, leaves, and peels oils of four sour orange provenances. Journal of Essential Oil Bearing Plants, 10, (6): 453-464. 2007.

[8] Cheong, M.-W., Loke, X.-Q., Liu, S.-Q., Pramudya, K., Curran, P., $\mathrm{Yu}, \mathrm{B}$. Characterization of volatile compounds and aroma profiles of malaysian pomelo (Citrus grandis (l.) Osbeck) blossom and peel. Journal of Essential Oil Research, 23, (2): 34-44. 2011.

[9] Chung, M. Volatile compounds of the hallabong (Citrus kiyomi $\times$ Citrus ponkan) blossom. Food Science and Biotechnology, 21, (1): 285-290. 2012

[10] Ferhat, M. A., Meklati, B. Y., Smadja, J., Chemat, F. An improved microwave clevenger apparatus for distillation of essential oils from orange peel. Journal of Chromatography A, 1112: (1-2), 121-126. 2006.

[11] Fanciullino, A.-L., Tomi, F., Luro, F., Desjobert, J. M., Casanova, J. Chemical variability of peel and leaf oils of mandarins. Flavour and Fragrance Journal, 21, (2): 359-367. 2006.

[12] Fisher, K., Phillips, C. Potential antimicrobial uses of essential oils in food: is citrus the answer? Trends in Food Science \& Technology, 19, (3): 156-164. 2008.

[13] Flamini, G., Tebano, M., Cioni, P. L. Volatiles emission patterns of different plant organs and pollen of citrus limon. Analytica Chimica Acta, 589, (1): 120-124. 2007.

[14] González-Mas, M. C., Rambla, J. L., Alamar, M. C., Gutiérrez, A., Granell, A. Comparative analysis of the volatile fraction of fruit juice from different citrus species. Plos One, 6, (7), e22016. 2011.

[15] Hosni, K., Zahed, N., Chrif, R., Abid, I., Medfei, W., Kallel, M. Brahim, N. B., Sebei, H. Composition of peel essential oils from four selected tunisian citrus species: evidence for the genotypic influence. Food Chemistry, 123, (4): 1098-1104. 2010.

[16] Hwang, S. L., Shih, P. H., Yen, G. C. Neuroprotective effects of citrus flavonoids. Journal of Agricultural and Food chemistry, 60, (4): 877-885. 2012.

[17] Jabalpurwala, F. A., Smoot, J. M.; Rouseff, R. L. A. comparison of citrus blossom volatiles. Phytochemistry, 70, (11-12): 14281434. 2009.

[18] Li, X.; Xie, R., Lu, Z., Zhou, Z. The origin of cultivated citrus as inferred from internal transcribed spacer and chloroplast DNA sequence and amplified fragment length polymorphism fingerprints. Journal of the American Society for Horticultural Science, 135, (4): 341-350. 2010.
[19] Liu, C., Jiang, D., Cheng, Y., Deng, X., Chen, F., Fang, L., Ma, Z., $\mathrm{Xu}$, J. Chemotaxonomic study of citrus, poncirus and fortunella genotypes based on peel oil volatile compounds - decipher- ing the genetic origin of mangshanyegan (Citrus nobilis Lauriro). Plos One, 8, (3), e58411. 2013.

[20] Lota, M. L., de Rocca Serra, D., Tomi, F., Joseph, C. Chemical variability of peel and leaf essential oils of mandarins from citrus reticulata blanco. Biochemical Systematics and Ecology, 28, (1): 61-78. 2000.

[21] Lu, Y., Zhang, C., Bucheli, P., Wei, D. Citrus flavonoids in fruit and traditional chinese medicinal food ingredients in China. Plant Foods for Human Nutrition, 61, (2): 55-63. 2006.

[22] Lu, Z. H., Zhou, Z. Q., Xie, R. J. Molecular phylogeny of the "true citrus fruit trees” group (Aurantioideae, Rutaceae) as inferred from chloroplast DNA sequence. Agricultural Sciences in China, 10, (1): 49-57. 2011.

[23] Malik, M. N., Scora, R. W., Soost, R. K. Studies in the origin of the lemon. [s.n.]: Berkeley, 1974.

[24] Miller, J., Thompson, P., Hakim, I., Chow, H. H. S., Thomson, C. D-Limonene: a bioactive food component from citrus and evidence for a potential role in breast cancer prevention and treatment. Oncology Reviews, 5, (1): 31-42. 2011.

[25] Nicolosi, E., Deng, Z. N., Gentile, A., La Malfa, S., Continella, G., Tribulato, E. Citrus phylogeny and genetic origin of important species as investigated by molecular markers. Theoretical and Applied Genetics, 100, (8): 1155-1166. 2000.

[26] Omori, H., Nakahara, K., Umano, K. Characterization of aroma compounds in the peel extract of Jabara (Citrus jabara Hort. ex Tanaka). Flavour and fragrance journal, 26, (6): 396-402. 2011.

[27] Ramadan, W., Mourad, B., Ibrahim, S., Sonbol, F. Oil of bitter orange: new topical antifungal agent. International Journal of Dermatology, 35, (6): 448-449. 1996.

[28] Rossi, Y. E., Palacios, S. M. Fumigant toxicity of Citrus sinensis essential oil on musca domestica L. adults in the absence and presence of a P450 inhibitor. Acta Tropica, 127, (1): 33-37. 2013.

[29] Sarrou, E., Chatzopoulou, P., Dimassi-Theriou, K., Therios, I. Volatile constituents and antioxidant activity of peel, flowers and leaf oils of citrus aurantium L. growing in Greece. Molecules, 18, (9): 10639-10647. 2013.

[30] Spiegel, R., Goldschmidt, E. E. Biology of citrus. Cambridge university press, 1996.

[31] Sumner, L., Huhman, D., Urbanczyk-Wochniak, E., Lei, Z. In Plant systems biology, Baginsky, S.; Fernie, A. Eds. Birkhäuser Basel. SpringerLink, 2007.

[32] Tripoli, E., Guardia, M. L., Giammanco, S., Majo, D. D., Giammanco, M. Citrus flavonoids: molecular structure, biological activity and nutritional properties: A review. Food Chemistry, 104, (2): 466-479. 2007.

[33] Vekiari, S. A., Protopapadakis, E. E., Papadopoulou, P., Papanicolaou, D., Panou, C., Vamvakias, M. Composition and seasonal variation of the essential oil from leaves and peel of a cretan lemon variety. Journal of Agricultural and Food Chemistry, 50, (1): 147-153. 2001.

[34] Wei, A., Shibamoto, T. Antioxidant/Lipoxygenase inhibitory activities and chemical compositions of selected essential oils. Journal of Agricultural and Food Chemistry, 58, (12): 7218-7225. 2010.

[35] Zhang, Y., Sun, Y., Xi, W., Shen, Y., Qiao, L., Zhong, L., Ye, X., Zhou, Z. Phenolic compositions and antioxidant capacities of Chinese wild mandarin (Citrus reticulata Blanco) fruits. Food Chemistry, 145, (1): 674-680. 2014. 